Article

\title{
Dual Hesitant Pythagorean Fuzzy Heronian Mean Operators in Multiple Attribute Decision Making
}

\author{
Mei Tang ${ }^{1}$, Jie Wang ${ }^{1}$, Jianping Lu ${ }^{1}$, Guiwu Wei ${ }^{1}\left(\mathbb{D}\right.$, Cun Wei $^{1,2}\left(\mathbb{D}\right.$ and Yu Wei ${ }^{3, *}$ \\ 1 School of Business, Sichuan Normal University, Chengdu 610101, China; 15882583803@163.com (M.T.); \\ JW970326@163.com (J.W.); lujp2002@163.com (J.L.); weiguiwu1973@sicnu.edu.cn (G.W.); \\ weicun1990@163.com (C.W.) \\ 2 School of Statistics, Southwestern University of Finance and Economics, Chengdu 611130, China \\ 3 School of Finance, Yunnan University of Finance and Economics, Kunming 650221, China \\ * Correspondence: weiyusy@126.com
}

Received: 27 March 2019; Accepted: 6 April 2019; Published: 10 April 2019

\begin{abstract}
On account of the indeterminacy and subjectivity of decision makers (DMs) in complexity decision-making environments, the evaluation information over alternatives presented by DMs is usually fuzzy and ambiguous. As the generalization of intuitionistic fuzzy sets (IFSs), the Pythagorean fuzzy set (PFS) is more useful in expressing fuzzy and ambiguous information. Meanwhile, in order to consider human hesitance, dual hesitant Pythagorean fuzzy sets (DHPFSs) are presented, which can be more valid for handling real multiple attribute decision-making (MADM) problems. To fuse the information in DHPFSs more effectively, in this article, some dual hesitant Pythagorean fuzzy Heronian mean operators, which can consider the relationships between arguments being fused, are defined and studied. Evidently, the new proposed operators can obtain more exact results than other existing methods. In addition, some important properties of these Heronian mean (HM) operators are discussed. Subsequently, the defined aggregation operators are used in MADM with dual hesitant Pythagorean fuzzy numbers (DHPFNs), and the MADM model is developed. In accordance with the defined operators and the built model, the dual hesitant Pythagorean fuzzy generalized weighted Heronian mean (DHPFGWHM) operator and dual hesitant Pythagorean fuzzy generalized geometric weighted Heronian mean (DHPFGGWHM) operator are applied to deal with the green supplier selection in supply chain management, and the availability and superiority of the proposed operators are analyzed by comparing them with some existing approaches. The method presented in this paper can effectively solve the MADM problems in which the decision-making information is expressed by DHPFNs and the attributes are interactive.
\end{abstract}

Keywords: multiple attribute decision-making (MADM); dual hesitant Pythagorean fuzzy sets (DHPFSs); dual hesitant Pythagorean fuzzy generalized weighted Heronian mean (DHPFGWHM) operator; dual hesitant Pythagorean fuzzy generalized geometric weighted Heronian mean (DHPFGGWHM) operator; green supplier selection; supply chain management

\section{Introduction}

In practical decision making environments, it is difficult for decision makers (DMs) to give evaluated information with exact real numbers. To overcome this disadvantage, Zadeh [1] has developed the fuzzy set (FS) theory which utilizes the function of membership degree to express decision making information instead of crisp results between 0 and 1. Based on studies of FS, Atanassov [2] further proposed another function, named the non-membership degree, as a supplementary function. Thus, the intuitionistic fuzzy set (IFS) was constructed; in the IFS each intuitionistic fuzzy set is characterized by the functions of membership degree and non-membership degree between 0 and 1 , 
and the sum of these are limited to 1 . Subsequent to these studies, more and more scholars have studied the IFS in relation to many multiple attribute decision making (MADM) problems [3-16]. Xu [17] has defined some intuitionistic fuzzy weighted average operators. $\mathrm{Xu}$ and Yager [18] have proposed some aggregation operators, such as the intuitionistic fuzzy weighted geometric (IFWG) operator, the intuitionistic fuzzy ordered weighted geometric (IFOWG) operator, and the intuitionistic fuzzy hybrid geometric (IFHG) operator, based on geometric operation laws and an intuitionistic fuzzy environment. Hung and Yang [19] studied the similarity measures of intuitionistic fuzzy sets based on an Lp-metric. Park et al. [20] have put forward some distance measures of interval-valued intuitionistic fuzzy sets. To handle intuitionistic fuzzy MADM problems with incomplete weight information, Wei [21] utilized the maximizing deviation method to build two intuitionistic fuzzy nonlinear programming models. By considering the entropy weight of an intuitionistic fuzzy set, Hung et al. [22] established a fuzzy TOPSIS decision making model. Luo [23] defined a projection method based on intuitionistic fuzzy information with uncertain attribute weights for MADM. Ye [24] has provided a cross-entropy method with which to handle decision making problems with interval-valued intuitionistic fuzzy information. On account of the indeterminacy of DMs, Zhang [25] has presented some interval-valued hesitant fuzzy aggregation operators and applied them to MADM problems. Liao and Xu [26] have defined some intuitionistic fuzzy hybrid weighted aggregation operators. To express fuzzy information more easily, Liu et al. [27] have developed the concepts of hesitant intuitionistic fuzzy linguistic elements (HLFLEs) and have defined some weighted aggregation operators. Peng et al. [28] have discussed the MADM approach under a hesitant interval-valued intuitionistic fuzzy environment. Chen and Huang [29] have given the definition of hesitant triangular intuitionistic fuzzy set (HTIFS) and investigated its applications in MADM problems.

In addition, some other fuzzy decision making approaches have been proposed by numerous scholars [30-33]. Hu et al. [34] have proposed a novel approach combining fuzzy data envelopment analysis (DEA) and the analytical hierarchical process (AHP) to rank units with multiple fuzzy criteria. Ziemba et al. [35] have studied the online comparison system with certain and uncertain criteria. Diouf and Kwak [36] have studied fuzzy AHP, DEA, and managerial analysis for supplier selection and development from the perspective of open innovation. Dong et al. [37] utilized the modified fuzzy VIKOR and scalable computing method to study the performance evaluation of residential demand responses. Kim and Kim [38] have developed a new model for the optimal LNG import portfolio. Chou et al. [39] have used fuzzy AHP and fuzzy TOPSIS to evaluate the human resource in science and technology (HRST) performance of Southeast Asian countries.

In addition, as an effective MADM tool, the Pythagorean fuzzy set (PFS) $[40,41]$ has emerged as a means to describe the indeterminacy and complexity of evaluation information. Similarly to the IFS, the PFS also consists of a membership degree and non-membership degree, the sum of the squares of which is restricted to 1 . Thus, it is clear that the PFS is more widespread than the IFS and can express more decision-making information. For instance, the membership is given as 0.6 and the non-membership is given as 0.8 ; it is obvious that this problem is only valid for the PFS. In other words, all intuitionistic fuzzy decision-making problems are a special case of Pythagorean fuzzy decision-making problems, which means that the PFS is more efficient in dealing with MADM problems. In previous literature, some research works have been studied by a large number of investigators. Zhang and $\mathrm{Xu}$ [42] defined the Pythagorean fuzzy TOPSIS model to deal with MADM problems. Peng and Yang [43] primarily proposed two Pythagorean fuzzy operations including the division and subtraction operations to better understand PFS. Reformat and Yager [44] handled the collaborative-based recommender system with Pythagorean fuzzy information. Gou et al. [45] have studied some important properties of continuous Pythagorean fuzzy information. Garg [46] has defined some new Pythagorean fuzzy aggregation operators, including Pthe ythagorean fuzzy Einstein weighted averaging (PFEWA) operator, the Pythagorean fuzzy Einstein ordered weighted averaging (PFEOWA) operator, the generalized Pythagorean fuzzy Einstein weighted averaging (GPFEWA) operator and the generalized Pythagorean fuzzy Einstein ordered weighted averaging (GPFEOWA) 
operator. Zeng et al. [47] have utilized the Pythagorean fuzzy ordered weighted averaging weighted average distance (PFOWAWAD) operator to study Pythagorean fuzzy MADM issues. Ren et al. [48] built a Pythagorean fuzzy TODIM model. Liang et al. [49] investigated some Bonferroni mean operators with Pythagorean fuzzy information. Liang et al. [50] have presented Pythagorean fuzzy Bonferroni mean aggregation operators based on geometric averaging (GA) operations. Combining PFSs [40,41] and dual hesitant fuzzy sets (DHFSs) [51,52], Wei and Lu [53] introduced a definition of dual hesitant Pythagorean fuzzy sets (DHPFSs) and proposed some dual hesitant Pythagorean fuzzy Hamacher aggregation operators. Obviously, the DHPFSs have the advantages of considering the hesitance of DMs and expressing fuzzy information more effectively and reasonably.

However, in practical MADM problems, some relationships do exist between arguments being fused, and it is obvious that the dual hesitant Pythagorean fuzzy Hamacher aggregation operators defined by Wei and $\mathrm{Lu}$ [53] do not take the relationships between the arguments being fused into consideration. Thus, it is necessary to find another more effective method with which to fuse dual hesitant Pythagorean fuzzy information. To date, the Heronian mean (HM) [54] operator, which can effectively take the interrelationship between arguments into account, has drawn a large quantity of scholars' attention [55-59]. Based on intuitionistic fuzzy information and a geometric operator, $\mathrm{Yu}$ [54] developed the intuitionistic fuzzy geometric Heronian mean (IFGHM) operator and the intuitionistic fuzzy geometric weighted Heronian mean (IFGWHM) operator. Liu et al. [60] further proposed some Heronian mean operators under an intuitionistic uncertain linguistic environment for MADM. Yu et al. [61] have defined some linguistic hesitant fuzzy Heronian mean (LHFHM) operators. Li et al. [62] extended the Heronian mean operator to a single valued neutrosophic environment. Wei et al. [63] have presented some q-rung orthopair Heronian mean operators. Considering linguistic variables, Li et al. [64] developed some q-rung orthopair linguistic Heronian mean operators.

In this paper, based on the generalized Heronian mean (GHM) operator and generalized geometric Heronian mean (GGHM) operator, we develop some dual hesitant Pythagorean fuzzy generalized Heronian mean aggregation operators. The remainder of this paper is set out as follows. In the next section, we introduce some basic concepts related to the Pythagorean fuzzy set (PFS), the dual hesitant Pythagorean fuzzy set (DHPFS), and their operational laws. In Section 3, we propose some dual hesitant Pythagorean fuzzy Heronian mean aggregation operators such as: the dual hesitant Pythagorean fuzzy generalized weighted Heronian mean (DHPFGWHM) operator and the dual hesitant Pythagorean fuzzy generalized geometric weighted Heronian mean (DHPFGGWHM) operator. In Section 4, based on the DHPFGWHM and DHPFGGWHM operators, we propose some models for MADM problems with dual hesitant Pythagorean fuzzy information. In Section 5, we present a numerical example for supplier selection in supply chain management with dual hesitant Pythagorean fuzzy information in order to illustrate the method proposed in this paper. Section 6 concludes the paper with some remarks.

\section{Preliminaries}

\subsection{Pythagorean Fuzzy Set}

The fundamental definition of PFSs $[40,41]$ are briefly introduced in this section. Then, novel score and accuracy functions of Pythagorean fuzzy numbers (PFNs) are developed. Furthermore, the comparison laws of PFNs are proposed.

Definition 1 [40,41]. Let X be a fixed set. A Pythagorean fuzzy set (PFS) is an object which can be denoted as

$$
P=\left\{\left\langle x,\left(\alpha_{P}(x), \beta_{P}(x)\right)\right\rangle \mid x \in X\right\}
$$

where the function $\alpha_{P}: X \rightarrow[0,1]$ indicates the degree of membership and the function $\beta_{P}: X \rightarrow[0,1]$ indicates the degree of non-membership of the element $x \in X$ to $P$, respectively, and, for each $x \in X$, it holds that

$$
\left(\alpha_{p}(x)\right)^{2}+\left(\beta_{p}(x)\right)^{2} \leq 1
$$


Definition 2 [42]. Assume that $p_{1}=\left(\alpha_{1}, \beta_{1}\right), p_{2}=\left(\alpha_{2}, \beta_{2}\right)$, and $p=(\alpha, \beta)$ are three Pythagorean fuzzy numbers (PFNs). Then, some basic operation laws of them can be expressed as:

$$
\begin{aligned}
& \text { (1) } p_{1} \oplus p_{2}=\left(\sqrt{\left(\alpha_{p_{1}}\right)^{2}+\left(\alpha_{p_{2}}\right)^{2}-\left(\alpha_{p_{1}}\right)^{2}\left(\alpha_{p_{1}}\right)^{2}}, \beta_{p_{1}} \beta_{p_{2}}\right) ; \\
& \text { (2) } p_{1} \otimes p_{2}=\left(\alpha_{p_{1}} \alpha_{p_{2}}, \sqrt{\left(\beta_{p_{1}}\right)^{2}+\left(\beta_{p_{2}}\right)^{2}-\left(\beta_{p_{1}}\right)^{2}\left(\beta_{p_{2}}\right)^{2}}\right) ; \\
& \text { (3) } \lambda p=\left(\sqrt{1-\left(1-\alpha^{2}\right)^{\lambda}}, \beta^{\lambda}\right), \lambda>0 ; \\
& \text { (4) }(p)^{\lambda}=\left(\alpha^{\lambda}, \sqrt{1-\left(1-\beta^{2}\right)^{\lambda}}\right), \lambda>0 ; \\
& \text { (5) } p^{c}=(\beta, \alpha) .
\end{aligned}
$$

Example 1. Assume that $p_{1}=(0.5,0.7), p_{2}=(0.3,0.4)$, and $p=(0.6,0.3)$ are three Pythagorean fuzzy numbers (PFNs). Suppose $\lambda=3$. Then, according to the above operation laws, we can obtain:

$$
\begin{aligned}
& \text { (1) } p_{1} \oplus p_{2}=\left(\sqrt{(0.5)^{2}+(0.3)^{2}-(0.5)^{2} \times(0.3)^{2}}, 0.7 \times 0.4\right)=(0.56,0.28) ; \\
& \text { (2) } p_{1} \otimes p_{2}=\left(0.5 \times 0.3, \sqrt{(0.7)^{2}+(0.4)^{2}-(0.7)^{2} \times(0.4)^{2}}\right)=(0.15,0.64) ; \\
& \text { (3) } 3 \times p=\left(\sqrt{1-\left(1-0.6^{2}\right)^{3}}, 0.3^{3}\right)=(0.8590,0.0270) ; \\
& \text { (4) }(p)^{3}=\left(0.6^{3}, \sqrt{1-\left(1-0.3^{2}\right)^{3}}\right)=(0.4964,0.2160) ; \\
& \text { (5) } p^{c}=(0.3,0.6) .
\end{aligned}
$$

\subsection{Dual Hesitant Pythagorean Fuzzy Set}

In this section, we shall introduce the basic definition of the dual hesitant Pythagorean fuzzy set (DHPFS), which is the generalization of the PFS [40,41] and the dual hesitant fuzzy set (DHFS) [51,52]. It is obvious that the DHPFSs consist of two parts, namely, the function of membership hesitancy and the function of non-membership hesitancy, which support more exemplary and flexible access to assigning values for each element in the domain, meaning we have to handle two kinds of hesitancy in this situation.

Definition 3 [53]. Assume that $X$ is a fixed set. Then, a dual hesitant Pythagorean fuzzy set (DHPFS) on $X$ can be developed as

$$
\widetilde{P}=\left(\left\langle x, h_{\widetilde{P}}(x), g_{\widetilde{P}}(x)\right\rangle \mid x \in X\right)
$$

in which $h_{\widetilde{P}}(x)$ and $g_{\widetilde{P}}(x)$ are two sets of some values in $[0,1]$, indicating that the function of membership degrees and non-membership degrees of the element $x \in X$ to the set $\widetilde{P}$, respectively, satisfies the condition

$$
\alpha^{2}+\beta^{2} \leq 1
$$

where $\alpha \in h_{\widetilde{p}}(x), \beta \in g_{\widetilde{p}}(x)$, for all $x \in X$. For convenience, the pair $\widetilde{p}(x)=\left(h_{\widetilde{p}}(x), g_{\widetilde{p}}(x)\right)$ is called a dual hesitant Pythagorean fuzzy number (DHPFN) denoted by $\widetilde{p}=(h, g)$, with the conditions $\alpha \in h, \beta \in g$, $0 \leq \alpha, \beta \leq 1,0 \leq \alpha^{2}+\beta^{2} \leq 1$. 
Definition 4 [53]. Let $\tilde{p}=(h, g)$ be a DHPFN. Then, $s(\widetilde{p})=\frac{1}{2}\left(1+\frac{1}{\# h} \sum_{\alpha \in h} \alpha^{2}-\frac{1}{\# g} \sum_{\beta \in g} \beta^{2}\right)$ is the score function of $\widetilde{p}$, and $H(\widetilde{p})=\frac{1}{\# h} \sum_{\alpha \in h} \alpha^{2}+\frac{1}{\# g} \sum_{\beta \in g} \beta^{2}$ is the accuracy function of $\widetilde{p}$, where \#h and \#g are the numbers of the elements in $h$ and $g$ respectively. Then, let $\widetilde{p}_{i}=\left(h_{i}, g_{i}\right)(i=1,2)$ be any two DHPFNs. Subsequently, we have the following comparison laws:

- If $s\left(\widetilde{p}_{1}\right)>s\left(\widetilde{p}_{2}\right)$, then $\widetilde{p}_{1}$ is superior to $\widetilde{p}_{2}$, denoted by $\widetilde{p}_{1}>\widetilde{p}_{2}$;

- If $s\left(\widetilde{p}_{1}\right)=s\left(\widetilde{p}_{2}\right)$, then:

(1) If $p\left(\widetilde{p}_{1}\right)=p\left(\widetilde{p}_{2}\right)$, then $\widetilde{p}_{1}$ is equivalent to $\widetilde{p}_{2}$, denoted by $\widetilde{p}_{1} \sim \widetilde{p}_{2}$;

(2) If $p\left(\widetilde{p}_{1}\right)>p\left(\widetilde{p}_{2}\right)$, then $\widetilde{p}_{1}$ is superior to $\widetilde{p}_{2}$, denoted by $\widetilde{p}_{1}>\widetilde{p}_{2}$.

Definition 5 [53]. Assume that $\widetilde{p}_{1}=\left(h_{1}, g_{1}\right), \widetilde{p}_{2}=\left(h_{2}, g_{2}\right)$, and $\widetilde{p}=(h, g)$ are three DHPFNs. Then, some basic operation laws of these can be expressed as:

(1) $\tilde{p}^{\lambda}=\cup_{\alpha \in h, \beta \in g}\left\{\left\{\alpha^{\lambda}\right\},\left\{\sqrt{1-\left(1-\beta^{2}\right)^{\lambda}}\right\}\right\}, \lambda>0$

(2) $\lambda \widetilde{p}=\cup_{\alpha \in h, \beta \in g}\left\{\left\{\sqrt{1-\left(1-\alpha^{2}\right)^{\lambda}}\right\},\left\{\beta^{\lambda}\right\}\right\}, \lambda>0$;

(3) $\widetilde{p}_{1} \oplus \widetilde{p}_{2}=\cup_{\alpha_{1} \in h_{1}, \alpha_{2} \in h_{2}, \beta_{1} \in g_{1}, \beta_{2} \in g_{2}}\left\{\left\{\sqrt{\left(\alpha_{1}\right)^{2}+\left(\alpha_{2}\right)^{2}-\left(\alpha_{1}\right)^{2}\left(\alpha_{2}\right)^{2}}\right\},\left\{\beta_{1} \beta_{2}\right\}\right\}$;

(4) $\widetilde{p}_{1} \otimes \widetilde{p}_{2}=\cup_{\alpha_{1} \in h_{1}, \alpha_{2} \in h_{2}, \beta_{1} \in g_{1}, \beta_{2} \in g_{2}}\left\{\left\{\alpha_{1} \alpha_{2}\right\},\left\{\sqrt{\left(\beta_{1}\right)^{2}+\left(\beta_{2}\right)^{2}-\left(\beta_{1}\right)^{2}\left(\beta_{2}\right)^{2}}\right\}\right\}$.

Example 2. Assume that $p_{1}=\{\{0.7\},\{0.3\}\}, p_{2}=\{\{0.1,0.2\},\{0.4\}\}$, and $p=\{\{0.5,0.6\},\{0.4\}\}$ are three Pythagorean fuzzy numbers. Suppose $\lambda=3$. Then, according to the above operation laws, we can obtain

(1) $\widetilde{p}^{3}=\cup_{\alpha \in h, \beta \in g}\left\{\left\{0.5^{3}, 0.6^{3}\right\},\left\{\sqrt{1-\left(1-0.4^{2}\right)^{3}}\right\}\right\}=\{\{0.125,0.216\},\{0.638\}\} ;$

(2) $3 \widetilde{p}=\cup_{\alpha \in h, \beta \in g}\left\{\left\{\begin{array}{l}\sqrt{1-\left(1-0.5^{2}\right)^{3}}, \\ \sqrt{1-\left(1-0.6^{2}\right)^{3}}\end{array}\right\},\left\{0.4^{3}\right\}\right\}=\{\{0.760,0.859\},\{0.064\}\}$;

(3) $\widetilde{p}_{1} \oplus \widetilde{p}_{2}=\cup_{\alpha_{1} \in h_{1}, \alpha_{2} \in h_{2}, \beta_{1} \in g_{1}, \beta_{2} \in g_{2}}\left\{\left\{\begin{array}{l}\sqrt{0.7^{2}+0.1^{2}-0.7^{2} \times 0.1^{2}}, \\ \sqrt{0.7^{2}+0.2^{2}-0.7^{2} \times 0.2^{2}}\end{array}\right\},\{0.3 \times 0.4\}\right\}$

$=\{\{0.704,0.714\},\{0.120\}\}$

(4) $\widetilde{p}_{1} \otimes \widetilde{p}_{2}=\cup_{\alpha_{1} \in h_{1}, \alpha_{2} \in h_{2}, \beta_{1} \in g_{1}, \beta_{2} \in g_{2}}\left\{\{0.7 \times 0.1,0.7 \times 0.2\},\left\{\sqrt{0.3^{2}+0.4^{2}-0.3^{2} \times 0.4^{2}}\right\}\right\}$ $=\{\{0.070,0.140\},\{0.485\}\}$

\subsection{The Heronian Mean Operator}

Definition 6 [65]. Let $b_{i}(i=1,2, \cdots, n)$ be a group of nonnegative real numbers. Then, the Heronian mean (HM) operator can be defined as:

$$
\operatorname{HM}\left(b_{1}, b_{2}, \ldots, b_{n}\right)=\frac{2}{n(n+1)} \sum_{i=1}^{n} \sum_{j=i}^{n}\left(b_{i} b_{j}\right)^{\frac{1}{2}}
$$


Definition 7 [54]. Assume that $\xi, \zeta>0$, and $b_{i}(i=1,2, \cdots, n)$ are a group of nonnegative real numbers. Then, the GHM operator can be defined as:

$$
\operatorname{GHM}^{\xi, \zeta}\left(a_{1}, a_{2}, \ldots, a_{n}\right)=\left(\frac{2}{n(n+1)} \sum_{i=1}^{n} \sum_{j=i}^{n} a_{i}^{\xi} a_{j}^{\zeta}\right)^{1 /(\xi+\zeta)}
$$

When $\xi=\zeta=1 / 2$, the GHM operator will reduce to the Heronian mean (HM) operator, which indicates that the HM operator is a special case of the GHM operator.

\section{Dual Hesitant Pythagorean Fuzzy Heronian Mean Operators}

In the following section $\mathrm{Xu}$ et al. [66] proposed the dual hesitant Pythagorean fuzzy generalized Heronian mean (DHPFGHM) operators based on dual hesitant Pythagorean fuzzy numbers (DHPFNs) and GHM operations. In addition, some important properties, such as idempotency, boundedness, and monotonicity are discussed.

\subsection{The DHPFGHM Aggregation Operator}

Definition 8 [66]. Let $\xi, \zeta>0$ and $\widetilde{p}_{j}=\left(h_{j}, g_{j}\right)(j=1,2, \cdots, n)$ be a group of DHPFNs. Then, we can define the DHPFGHM operator as

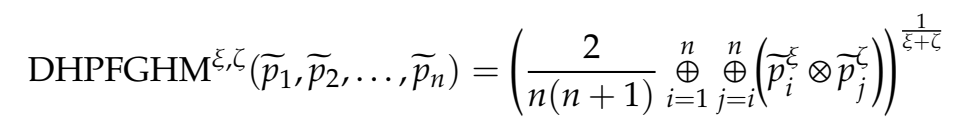

where " $\oplus$ " indicates the addition operation law and " $\otimes$ " indicates the multiplication operation law of the DHPFNs described in Definition 5. Then, according to these operation laws, Xu et al. [66] obtained Theorem 1.

Theorem 1 [66]. Let $\xi, \zeta>0$ and $\widetilde{p}_{j}=\left(h_{j}, g_{j}\right)(j=1,2, \cdots, n)$ be a group of dual hesitant Pythagorean fuzzy numbers, meaning their fused results by utilizing the DHPFGHM operator is also a DHPFN, and

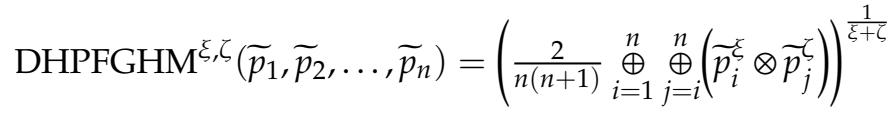

$$
\begin{aligned}
& =\cup_{\alpha_{i} \in h_{i}, \alpha_{j} \in h_{j}, \beta_{i} \in g_{i}, \alpha_{j} \in h_{j}}\left\{\begin{array}{l}
\left\{\left(\sqrt{1-\prod_{i=1, j=i}^{n}\left(1-\alpha_{i}^{2 \xi} \alpha_{j}^{2 \zeta}\right)^{\frac{2}{n(n+1)}}}\right)^{\frac{1}{\xi+\zeta}}\right\}, \\
\left\{\sqrt{1-\left(1-\prod_{i=1, j=i}^{n}\left(1-\left(1-\beta_{i}^{2}\right)^{\xi}\left(1-\beta_{j}^{2}\right)^{\zeta}\right)^{\frac{2}{n(n+1)}}\right)^{\frac{1}{\xi+\zeta}}}\right\}
\end{array}\right\}
\end{aligned}
$$

Proof. Based on Definition 5:

$$
\begin{aligned}
& \vec{p}_{i}^{\xi}=\cup_{\alpha_{i} \in h_{i}, \beta_{i} \in g_{i}}\left\{\left\{\alpha_{i}^{\xi}\right\},\left\{\sqrt{1-\left(1-\beta_{i}^{2}\right)^{\xi}}\right\}\right\} \\
& \vec{p}_{j}^{\zeta}=\cup_{\alpha_{j} \in h_{j}, \beta_{j} \in g_{j}}\left\{\left\{\alpha_{j}^{\zeta}\right\},\left\{\sqrt{1-\left(1-\beta_{j}^{2}\right)^{\zeta}}\right\}\right\}
\end{aligned}
$$


Thus,

$$
\widetilde{p}_{i}^{\xi} \otimes \widetilde{p}_{j}^{\zeta}=\cup_{\alpha_{i} \in h_{i}, \alpha_{j} \in h_{j}, \beta_{i} \in g_{i}, \alpha_{j} \in h_{j},}\left\{\left\{\alpha_{i}^{\xi} \alpha_{j}^{\zeta}\right\},\left\{\sqrt{1-\left(1-\beta_{i}^{2}\right)^{\xi}\left(1-\beta_{j}^{2}\right)^{\zeta}}\right\}\right\}
$$

Therefore,

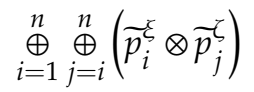

$$
\begin{aligned}
& =\cup_{\alpha_{i} \in h_{i}, \alpha_{j} \in h_{j}, \beta_{i} \in g_{i}, \alpha_{j} \in h_{j}}\left\{\begin{array}{l}
\left\{\sqrt{1-\prod_{i=1, j=i}^{n}\left(1-\alpha_{i}^{2 \xi} \alpha_{j}^{2 \zeta}\right)}\right\}, \\
\left\{\sqrt{\prod_{i=1, j=i}^{n}\left(1-\left(1-\beta_{i}^{2}\right)^{\xi}\left(1-\beta_{j}^{2}\right)^{\zeta}\right)}\right\}
\end{array}\right\}
\end{aligned}
$$

Furthermore,

$$
\begin{aligned}
& \frac{2}{n(n+1)} \underset{i=1}{\oplus} \bigoplus_{j=i}^{n}\left(\widetilde{p}_{i}^{\xi} \otimes \widetilde{p}_{j}^{\zeta}\right) \\
& =\cup_{\alpha_{i} \in h_{i}, \alpha_{j} \in h_{j}, \beta_{i} \in g_{i}, \alpha_{j} \in h_{j}}\left\{\begin{array}{l}
\left\{\sqrt{1-\prod_{i=1, j=i}^{n}\left(1-\alpha_{i}^{2 \xi} \alpha_{j}^{2 \zeta}\right)^{\frac{2}{n(n+1)}}}\right\}, \\
\left\{\sqrt{\prod_{i=1, j=i}^{n}\left(1-\left(1-\beta_{i}^{2}\right)^{\xi}\left(1-\beta_{j}^{2}\right)^{\zeta}\right)^{\frac{2}{n(n+1)}}}\right\}
\end{array}\right\}
\end{aligned}
$$

Therefore,

$$
\begin{aligned}
& \operatorname{DHPFGHM}^{\xi, \zeta}\left(\widetilde{p}_{1}, \widetilde{p}_{2}, \ldots, \widetilde{p}_{n}\right)=\left(\frac{2}{n(n+1)}{\left.\underset{i=1}{\oplus} \oplus_{j=i}^{n} \oplus_{i}^{n}\left(\vec{p}_{i}^{\xi} \otimes \widetilde{p}_{j}^{\zeta}\right)\right)^{\frac{1}{\xi+\zeta}}}\left\{\begin{array}{l}
\left\{\left(\sqrt{1-\prod_{i=1, j=i}^{n}\left(1-\alpha_{i}^{2 \xi} \alpha_{j}^{2 \zeta}\right)^{\frac{2}{n(n+1)}}}\right)^{\frac{1}{\xi+\zeta}}\right\}, \\
=\cup_{\alpha_{i} \in h_{i}, \alpha_{j} \in h_{j}, \beta_{i} \in g_{i}, \alpha_{j} \in h_{j}}\left\{\sqrt{1-\left(1-\prod_{i=1, j=i}^{n}\left(1-\left(1-\beta_{i}^{2}\right)^{\xi}\left(1-\beta_{j}^{2}\right)^{\zeta}\right)^{\frac{2}{n(n+1)}}\right)^{\frac{1}{\xi+\zeta}}}\right\}
\end{array}\right\}\right.
\end{aligned}
$$

Thus, the proof has been finished.

Example 3. Assume that $\widetilde{p}_{1}=\{\{0.7,0.8\},\{0.4\}\}, \widetilde{p}_{2}=\{\{0.3\},\{0.6,0.7\}\}, \widetilde{p}_{3}=\{\{0.1,0.3\},\{0.4,0.6\}\}$ and $\widetilde{p}_{4}=\{\{0.5\},\{0.5\}\}$ are four DHPFNs, and suppose that $\xi=2, \zeta=3$. Then according to the DHPFGHM operator, we can obtain the fused results as follows. For the membership degree function $\alpha$, the fused results are shown as:

$$
\begin{aligned}
& \alpha_{1}=\operatorname{DHPFGHM}{ }^{2,3}(0.7,0.3,0.1,0.5)=\left(\sqrt{1-\prod_{i=1, j=i}^{n}\left(1-\alpha_{i}^{2 \xi} \alpha_{j}^{2 \zeta}\right)^{\frac{2}{n(n+1)}}}\right)^{\frac{1}{\xi+\zeta}} \\
& =\left(\sqrt{1-\left(\begin{array}{l}
\left(1-0.7^{2 \times 2} \times 0.7^{2 \times 3}\right) \times\left(1-0.7^{2 \times 2} \times 0.3^{2 \times 3}\right) \times\left(1-0.7^{2 \times 2} \times 0.1^{2 \times 3}\right) \\
\times\left(1-0.7^{2 \times 2} \times 0.5^{2 \times 3}\right) \times\left(1-0.3^{2 \times 2} \times 0.3^{2 \times 3}\right) \times\left(1-0.3^{2 \times 2} \times 0.1^{2 \times 3}\right) \\
\times\left(1-0.3^{2 \times 2} \times 0.5^{2 \times 3}\right) \times\left(1-0.1^{2 \times 2} \times 0.1^{2 \times 3}\right) \times\left(1-0.1^{2 \times 2} \times 0.5^{2 \times 3}\right) \\
\times\left(1-0.5^{2 \times 2} \times 0.5^{2 \times 3}\right)
\end{array}\right)^{\frac{1}{10}}}\right)^{\frac{1}{2+3}} \\
& =0.5658
\end{aligned}
$$


Similarly, we can obtain

$$
\begin{aligned}
& \alpha_{2}=\operatorname{DHPFGHM}^{2,3}(0.7,0.3,0.3,0.5)=0.5664 \\
& \alpha_{3}=\operatorname{DHPFGHM}^{2,3}(0.8,0.3,0.1,0.5)=0.6492 \\
& \alpha_{4}=\operatorname{DHPFGHM}^{2,3}(0.8,0.3,0.3,0.5)=0.6432
\end{aligned}
$$

Hence, we can get $\alpha=\{0.5658,0.5664,0.6429,0.6432\}$.

For the non-membership degree function $\beta$, the fused results are shown as:

$$
\begin{aligned}
& \beta_{1}=\operatorname{DHPFGHM}^{2,3}(0.4,0.6,0.4,0.5)=\sqrt{1-\left(1-\prod_{i=1, j=i}^{n}\left(1-\left(1-\beta_{i}^{2}\right)^{\xi}\left(1-\beta_{j}^{2}\right)^{\zeta}\right)^{\frac{2}{(n+1)}}\right)^{\frac{1}{\xi+\zeta}}} \\
& =\sqrt{\left.\left(\begin{array}{c}
\left(1-\left(1-0.4^{2}\right)^{2} \times\left(1-0.4^{2}\right)^{3}\right) \times\left(1-\left(1-0.4^{2}\right)^{2} \times\left(1-0.6^{2}\right)^{3}\right) \\
\times\left(1-\left(1-0.4^{2}\right)^{2} \times\left(1-0.4^{2}\right)^{3}\right) \times\left(1-\left(1-0.4^{2}\right)^{2} \times\left(1-0.5^{2}\right)^{3}\right) \\
\times\left(1-\left(1-0.6^{2}\right)^{2} \times\left(1-0.6^{2}\right)^{3}\right) \times\left(1-\left(1-0.6^{2}\right)^{2} \times\left(1-0.4^{2}\right)^{3}\right) \\
\times\left(1-\left(1-0.6^{2}\right)^{2} \times\left(1-0.5^{2}\right)^{3}\right) \times\left(1-\left(1-0.4^{2}\right)^{2} \times\left(1-0.4^{2}\right)^{3}\right) \\
\times\left(1-\left(1-0.4^{2}\right)^{2} \times\left(1-0.5^{2}\right)^{3}\right) \times\left(1-\left(1-0.5^{2}\right)^{2} \times\left(1-0.5^{2}\right)^{3}\right)
\end{array}\right)^{\frac{1}{10}}\right)^{\frac{1}{2+3}}} \\
& =0.4698
\end{aligned}
$$

Similarly, we can obtain

$$
\begin{aligned}
& \beta_{2}=\operatorname{DHPFGHM}^{2,3}(0.4,0.6,0.6,0.5)=0.5211 \\
& \beta_{3}=\operatorname{DHPFGHM}^{2,3}(0.4,0.7,0.4,0.5)=0.4856 \\
& \beta_{4}=\operatorname{DHPFGHM}^{2,3}(0.4,0.7,0.6,0.5)=0.5383
\end{aligned}
$$

Hence, we can get $\beta=\{0.4698,0.5211,0.4856,0.5383\}$.Therefore,

$$
\operatorname{DHPFGHM}\left(\widetilde{p}_{1}, \widetilde{p}_{2}, \widetilde{p}_{3}, \widetilde{p}_{4}\right)=\left\{\begin{array}{l}
\{0.5658,0.5664,0.6429,0.6432\} \\
\{0.4698,0.5211,0.4856,0.5383\}
\end{array}\right\} .
$$

It can be easily proven that the DHPFGHM operator satisfies the following properties.

Property 1. (Idempotency) If all $\tilde{p}_{j}=\left(h_{j}, g_{j}\right)(j=1,2, \cdots, n)$ are equal, i.e., $\tilde{p}_{j}=\widetilde{p}$ for all $j$, then

$$
\operatorname{DHPFGHM}^{\xi, \zeta}\left(\widetilde{p}_{1}, \widetilde{p}_{2}, \ldots, \widetilde{p}_{n}\right)=\widetilde{p}
$$

Property 2. (Boundedness) Let $\widetilde{p}_{j}=\left(h_{j}, g_{j}\right)(j=1,2, \cdots, n)$ be a collection of DHPFNs, and let

$$
\widetilde{p}^{+}=\cup_{\alpha_{j} \in h_{j}, \beta_{j} \in g_{j}}\left\{\left\{\max _{i}\left(\alpha_{i}\right)\right\},\left\{\min _{i}\left(\beta_{i}\right)\right\}\right\}, \widetilde{p}^{-}=\cup_{\alpha_{j} \in h_{j}, \beta_{j} \in g_{j}}\left\{\left\{\min _{i}\left(\alpha_{i}\right)\right\},\left\{\max _{i}\left(\beta_{i}\right)\right\}\right\}
$$

Then

$$
\widetilde{p}^{-} \leq \operatorname{DHPFGHM}^{\xi, \zeta}\left(\widetilde{p}_{1}, \widetilde{p}_{2}, \ldots, \widetilde{p}_{n}\right) \leq \widetilde{p}^{+}
$$


Property 3. (Monotonicity) Let $\widetilde{p}_{j}=\left(h_{j}, g_{j}\right)$ and $\widetilde{p}_{j}^{\prime}=\left(h_{j^{\prime}}^{\prime} g_{j}^{\prime}\right), j=1,2, \cdots, n$, be two sets of DHPFNs. If $\widetilde{p}_{j} \leq \widetilde{p}_{j}^{\prime}$, for all $j$, then

$$
\operatorname{DHPFGHM}^{\xi, \zeta}\left(\widetilde{p}_{1}, \widetilde{p}_{2}, \ldots, \widetilde{p}_{n}\right) \leq \operatorname{DHPFGHM}^{\xi, \zeta}\left(\widetilde{p}_{1}, \widetilde{p}_{2}, \ldots, \widetilde{p}_{n}^{\prime}\right)
$$

\subsection{The DHPFGWHM Aggregation Operator}

Using Definition 8, we can conclude that the DHPFGHM operator didn't take the importance of arguments being fused into account. However, in many practical MADM problems, we should consider the weights of attributes. To overcome this limitation of the DHPFGHM operator, we propose a novel DHPFGWHM operator as follows.

Definition 9. Assume that $\xi, \zeta>0$ and $\widetilde{p}_{j}=\left(h_{j}, g_{j}\right)(j=1,2, \cdots, n)$ are a group of dual hesitant Pythagorean fuzzy numbers (DHPFNs). Then, we define the DHPFGWHM operator as follows:

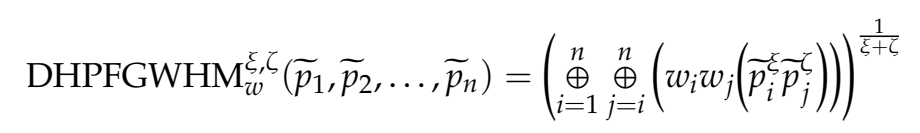

According to the operation laws of the DHPFNs described in Definition 5, we can obtain Theorem 2.

Theorem 2. Assume that $\xi, \zeta>0$ and $\widetilde{p}_{j}=\left(h_{j}, g_{j}\right)(j=1,2, \cdots, n)$ are a collection of DHPFNs with the weighting vector $w=\left(w_{1}, w_{2}, \ldots, w_{n}\right)^{T}$, which satisfies $w_{j}>0, i=1,2, \ldots, n$ and $\sum_{j=1}^{n} w_{j}=1$. Then, their fused result obtained by utilizing the DHPFGWHM operator is also a DHPFN, and

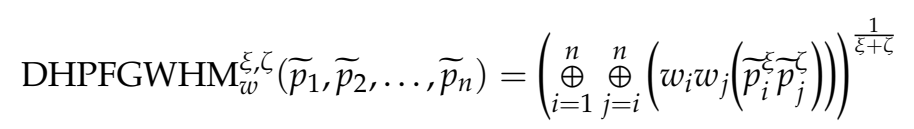

$$
\begin{aligned}
& =\cup_{\alpha_{i} \in h_{i}, \alpha_{j} \in h_{j}, \beta_{i} \in g_{i}, \alpha_{j} \in h_{j},}\left\{\begin{array}{l}
\left\{\left(\sqrt{1-\prod_{i=1, j=i}^{n}\left(1-\alpha_{i}^{2 \xi} \alpha_{j}^{2 \zeta}\right)^{w_{i} w_{j}}}\right)^{\frac{1}{\xi+\zeta}}\right\}, \\
\left\{\sqrt{1-\left(1-\prod_{i=1, j=i}^{n}\left(1-\left(1-\beta_{i}^{2}\right)^{\xi}\left(1-\beta_{j}^{2}\right)^{\zeta}\right)^{w_{i} w_{j}}\right)^{\frac{1}{\xi+\zeta}}}\right\}
\end{array}\right\}
\end{aligned}
$$

Proof. Based on Definition 5, we can obtain:

$$
\begin{aligned}
& \widetilde{p}_{i}^{\zeta}=\cup_{\alpha_{i} \in h_{i}, \beta_{i} \in g_{i}}\left\{\left\{\alpha_{i}^{\xi}\right\},\left\{\sqrt{1-\left(1-\beta_{i}^{2}\right)^{\xi}}\right\}\right\} \\
& \widetilde{p}_{j}^{\zeta}=\cup_{\alpha_{j} \in h_{j}, \beta_{j} \in g_{j}}\left\{\left\{\alpha_{j}^{\zeta}\right\},\left\{\sqrt{1-\left(1-\beta_{j}^{2}\right)^{\zeta}}\right\}\right\}
\end{aligned}
$$

Thus,

$$
\widetilde{p}_{i}^{\xi} \widetilde{p}_{j}^{\zeta}=\cup_{\alpha_{i} \in h_{i}, \alpha_{j} \in h_{j}, \beta_{i} \in g_{i}, \alpha_{j} \in h_{j},}\left\{\left\{\alpha_{i}^{\xi} \alpha_{j}^{\zeta}\right\},\left\{\sqrt{1-\left(1-\beta_{i}^{2}\right)^{\xi}\left(1-\beta_{j}^{2}\right)^{\zeta}}\right\}\right\}
$$


Therefore,

$$
\begin{aligned}
& w_{i} w_{j}\left(\widetilde{p}_{i}^{\xi} \widetilde{p}_{j}^{\zeta}\right) \\
& =\cup_{\alpha_{i} \in h_{i}, \alpha_{j} \in h_{j}, \beta_{i} \in g_{i}, \alpha_{j} \in h_{j},}\left\{\begin{array}{l}
\left\{\sqrt{1-\left(1-\alpha_{i}^{2 \xi} \alpha_{j}^{2 \zeta}\right)^{w_{i} w_{j}}}\right\}, \\
\left\{\left(\sqrt{\left.1-\left(1-\beta_{i}^{2}\right)^{\xi}\left(1-\beta_{j}^{2}\right)^{\zeta}\right)^{w_{i} w_{j}}}\right\}\right.
\end{array}\right\}
\end{aligned}
$$

Thereafter,

$$
\begin{aligned}
& \oplus_{i=1}^{n} \stackrel{\oplus}{j=i}^{n}\left(w_{i} w_{j}\left(\vec{p}_{i}^{\xi} \vec{p}_{j}^{\zeta}\right)\right) \\
& =\cup_{\alpha_{i} \in h_{i}, \alpha_{j} \in h_{j}, \beta_{i} \in g_{i}, \alpha_{j} \in h_{j},}\left\{\begin{array}{l}
\left\{\sqrt{1-\prod_{i=1, j=i}^{n}\left(1-\alpha_{i}^{2 \xi} \alpha_{j}^{2 \zeta}\right)^{w_{i} w_{j}}}\right\}^{\prime} \\
\left\{\left(\sqrt{\prod_{i=1, j=i}^{n}\left(1-\left(1-\beta_{i}^{2}\right)^{\xi}\left(1-\beta_{j}^{2}\right)^{\zeta}\right)}\right)^{w_{i} w_{j}}\right\}
\end{array}\right\}
\end{aligned}
$$

Therefore,

$$
\begin{aligned}
& \operatorname{DHPFGWHM}_{w}^{\xi, \zeta}\left(\widetilde{p}_{1}, \widetilde{p}_{2}, \ldots, \widetilde{p}_{n}\right)=\left(\bigoplus_{i=1}^{n} \bigoplus_{j=i}^{n}\left(w_{i} w_{j}\left(\widetilde{p}_{i}^{\xi} \widetilde{p}_{j}^{\zeta}\right)\right)\right)^{\frac{1}{\xi+\zeta}}
\end{aligned}
$$

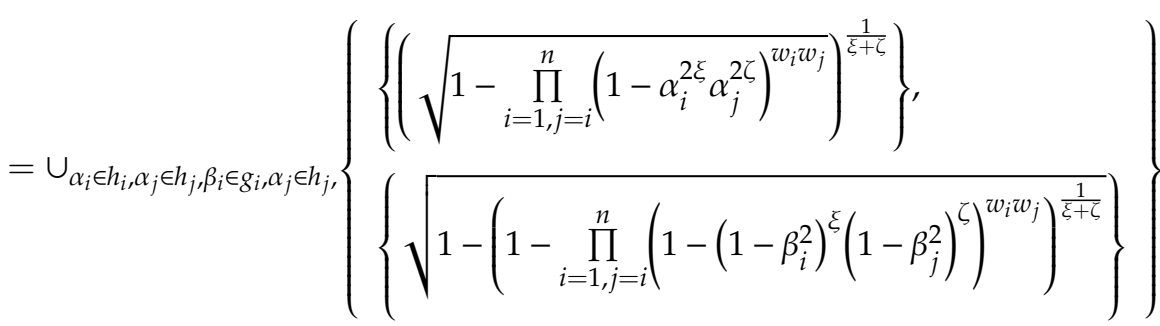

Thus, we have finished the proof.

Example 4. Assume that $\widetilde{p}_{1}=\{\{0.7,0.8\},\{0.4\}\}, \widetilde{p}_{2}=\{\{0.3\},\{0.6,0.7\}\}, \widetilde{p}_{3}=\{\{0.1,0.3\},\{0.4,0.6\}\}$ and $\tilde{p}_{4}=\{\{0.5\},\{0.5\}\}$ are four DHPFNs, and suppose that $\xi=2, \zeta=3$ and $w_{j}=(0.3,0.2,0.1,0.4)$. Then, according to the DHPFGWHM operator, we can obtain the fused results as follows. For the membership degree function $\alpha$, the fused results are shown as:

$$
\begin{aligned}
& \alpha_{1}=\operatorname{DHPFGWHM}^{2,3}(0.7,0.3,0.1,0.5)=\left(\sqrt{1-\prod_{i=1, j=i}^{n}\left(1-\alpha_{i}^{2 \xi} \alpha_{j}^{2 \zeta}\right)^{w_{i} w_{j}}}\right)^{\frac{1}{\xi+\zeta}} \\
& =\left(\begin{array}{l}
\begin{array}{l}
1-\left(1-0.7^{2 \times 2} \times 0.7^{2 \times 3}\right)^{0.3 \times 0.3} \times\left(1-0.7^{2 \times 2} \times 0.3^{2 \times 3}\right)^{0.3 \times 0.2} \times\left(1-0.7^{2 \times 2} \times 0.1^{2 \times 3}\right)^{0.3 \times 0.1} \\
\times\left(1-0.7^{2 \times 2} \times 0.5^{2 \times 3}\right)^{0.3 \times 0.4} \times\left(1-0.3^{2 \times 2} \times 0.3^{2 \times 3}\right)^{0.2 \times 0.2} \times\left(1-0.3^{2 \times 2} \times 0.1^{2 \times 3}\right)^{0.2 \times 0.1} \\
\times\left(1-0.3^{2 \times 2} \times 0.5^{2 \times 3}\right)^{0.2 \times 0.4} \times\left(1-0.1^{2 \times 2} \times 0.1^{2 \times 3}\right)^{0.1 \times 0.1} \times\left(1-0.1^{2 \times 2} \times 0.5^{2 \times 3}\right)^{0.1 \times 0.4} \\
\times\left(1-0.5^{2 \times 2} \times 0.5^{2 \times 3}\right)^{0.4 \times 0.4}
\end{array}
\end{array}\right. \\
& =0.5630
\end{aligned}
$$


Similarly, we can obtain

$$
\begin{aligned}
& \alpha_{2}=\operatorname{DHPFGWHM}^{2,3}(0.7,0.3,0.3,0.5)=0.5632 \\
& \alpha_{3}=\operatorname{DHPFGWHM}^{2,3}(0.8,0.3,0.1,0.5)=0.6376 \\
& \alpha_{4}=\operatorname{DHPFGWHM}^{2,3}(0.8,0.3,0.3,0.5)=0.6377
\end{aligned}
$$

Hence, we can get $\alpha=\{0.5630,0.5632,0.6376,0.6377\}$.

For the non-membership degree function $\beta$, the fused results are shown as:

$$
\begin{aligned}
& \beta_{1}=\operatorname{DHPFGWHM}^{2,3}(0.4,0.6,0.4,0.5)=\sqrt{1-\left(1-\prod_{i=1, j=i}^{n}\left(1-\left(1-\beta_{i}^{2}\right)^{\xi}\left(1-\beta_{j}^{2}\right)^{\zeta}\right)^{w_{i} w_{j}}\right)^{\frac{1}{\xi+\zeta}}} \\
& =\sqrt{\left(\begin{array}{l}
1-\left(1-\left(1-0.4^{2}\right)^{2} \times\left(1-0.4^{2}\right)^{3}\right)^{0.3 \times 0.3} \times\left(1-\left(1-0.4^{2}\right)^{2} \times\left(1-0.6^{2}\right)^{3}\right)^{0.3 \times 0.2} \\
\times\left(1-\left(1-0.4^{2}\right)^{2} \times\left(1-0.4^{2}\right)^{3}\right)^{0.3 \times 0.1} \times\left(1-\left(1-0.4^{2}\right)^{2} \times\left(1-0.5^{2}\right)^{3}\right)^{0.3 \times 0.4} \\
\times\left(1-\left(1-0.6^{2}\right)^{2} \times\left(1-0.6^{2}\right)^{3}\right)^{0.2 \times 0.2} \times\left(1-\left(1-0.6^{2}\right)^{2} \times\left(1-0.4^{2}\right)^{3}\right)^{0.2 \times 0.1} \\
\times\left(1-\left(1-0.6^{2}\right)^{2} \times\left(1-0.5^{2}\right)^{3}\right)^{0.2 \times 0.4} \times\left(1-\left(1-0.4^{2}\right)^{2} \times\left(1-0.4^{2}\right)^{3}\right)^{0.1 \times 0.1} \\
\times\left(1-\left(1-0.4^{2}\right)^{2} \times\left(1-0.5^{2}\right)^{3}\right)^{0.1 \times 0.4} \times\left(1-\left(1-0.5^{2}\right)^{2} \times\left(1-0.5^{2}\right)^{3}\right)^{0.4 \times 0.4}
\end{array}\right.} \\
& =0.5333
\end{aligned}
$$

Similarly, we can obtain

$$
\begin{aligned}
& \beta_{2}=\text { DHPFGWHM }^{2,3}(0.4,0.6,0.6,0.5)=0.5480 \\
& \beta_{3}=\operatorname{DHPFGWHM}^{2,3}(0.4,0.7,0.4,0.5)=0.5438 \\
& \beta_{4}=\operatorname{DHPFGWHM}^{2,3}(0.4,0.7,0.6,0.5)=0.5586
\end{aligned}
$$

Hence, we can get $\beta=\{0.5333,0.5480,0.5438,0.5586\}$. Therefore,

$$
\operatorname{DHPFGWHM}\left(\widetilde{p}_{1}, \widetilde{p}_{2}, \widetilde{p}_{3}, \widetilde{p}_{4}\right)=\left\{\begin{array}{c}
\{0.5630,0.5632,0.6376,0.6377\}, \\
\{0.5333,0.5480,0.5438,0.5586\}
\end{array}\right\} .
$$

It can be easily proven that the DHPFGWHM operator satisfies the following properties.

Property 4. (Idempotency) If all $\widetilde{p}_{j}=\left(h_{j}, g_{j}\right)(j=1,2, \cdots, n)$ are equal, i.e., $\widetilde{p}_{j}=\widetilde{p}$ for all $j$, then

$$
\operatorname{DHPFGWHM}_{w}^{\xi, \zeta}\left(\widetilde{p}_{1}, \widetilde{p}_{2}, \ldots, \widetilde{p}_{n}\right)=\widetilde{p}
$$

Property 5. (Boundedness) Let $\widetilde{p}_{j}=\left(h_{j}, g_{j}\right)(j=1,2, \cdots, n)$ be a collection of DHPFNs, and let

$$
\widetilde{p}^{+}=\cup_{\alpha_{j} \in h_{j}, \beta_{j} \in g_{j}}\left\{\left\{\max _{i}\left(\alpha_{i}\right)\right\},\left\{\min _{i}\left(\beta_{i}\right)\right\}\right\}, \widetilde{p}^{-}=\cup_{\alpha_{j} \in h_{j}, \beta_{j} \in g_{j}}\left\{\left\{\min _{i}\left(\alpha_{i}\right)\right\},\left\{\max _{i}\left(\beta_{i}\right)\right\}\right\}
$$

Then

$$
\widetilde{p}^{-} \leq \operatorname{DHPFGWHM}_{w}^{\xi, \zeta}\left(\widetilde{p}_{1}, \widetilde{p}_{2}, \ldots, \widetilde{p}_{n}\right) \leq \widetilde{p}^{+}
$$


Property 6. (Monotonicity) Let $\widetilde{p}_{j}=\left(h_{j}, g_{j}\right)$ and $\widetilde{p}_{j}^{\prime}=\left(h_{j^{\prime}}^{\prime}, g_{j}^{\prime}\right), j=1,2, \cdots, n$, be two sets of DHPFNs. If $\widetilde{p}_{j} \leq \widetilde{p}_{j}^{\prime}$, for all $j$, then

$$
\operatorname{DHPFGWHM}_{w}^{\xi, \zeta}\left(\widetilde{p}_{1}, \widetilde{p}_{2}, \ldots, \widetilde{p}_{n}\right) \leq \operatorname{DHPFGWHM}_{w}^{\xi, \zeta}\left(\vec{p}_{1}, \vec{p}_{2}, \ldots, \widetilde{p}_{n}\right)
$$

\subsection{The DHPFGGHM Aggregation Operator}

In the following, based on the geometric mean (GM) operator, Yu [54] extended the GHM operator to a GGHM operator which can be depicted as follows.

Definition 10 [54]. Assume that $\xi, \zeta>0$ and $b_{i}(i=1,2, \cdots, n)$ are a group of non-negative real numbers. Then, the generalizeGGHM) operator can be expressed as:

$$
\operatorname{GHM}^{\xi, \zeta}\left(a_{1}, a_{2}, \cdots, a_{n}\right)=\frac{1}{\xi+\zeta}\left(\prod_{i=1, j=i}^{n}\left(\xi a_{i}+\zeta a_{j}\right)\right)^{\frac{2}{n(n+1)}}
$$

In this section, we introduced the GGHM operator with dual hesitant Pythagorean fuzzy information. According to Definition 5, Xu et al. [66] gave the definition of the DHPFGGHM operator as follows.

Definition 11 [66]. Assume that $\xi, \zeta>0$ and $\widetilde{p}_{j}=\left(h_{j}, g_{j}\right)(j=1,2, \cdots, n)$ are a collection of DHPFNs. Then, the DHPFGGHM operator can be defined as:

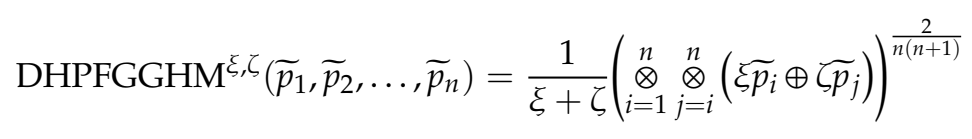

According to the operation laws of the DHPFNs described in Definition 5, Xu et al. [66] obtained Theorem 3.

Theorem 3 [66]. Assume that $\xi, \zeta>0$ and $\widetilde{p}_{j}=\left(h_{j}, g_{j}\right)(j=1,2, \cdots, n)$ are a group of DHPFNs. Then, their fused results obtained by utilizing the DHPFGGHM operator is also a DHPFN, and

$$
\left.\begin{array}{l}
\operatorname{DHPFGGHM}^{\xi, \zeta}\left(\widetilde{p}_{1}, \widetilde{p}_{2}, \ldots, \widetilde{p}_{n}\right)=\frac{1}{\xi+\zeta}\left(\prod_{i=1}^{n} \stackrel{\otimes}{j=i}_{i}^{n}\left(\xi \widetilde{p}_{i} \oplus \widetilde{p}_{j}\right)\right)^{\frac{2}{n(n+1)}} \\
=\cup_{\alpha_{i} \in h_{i}, \alpha_{j} \in h_{j}, \beta_{i} \in g_{i}, \alpha_{j} \in h_{j}}\left\{\sqrt{1-\left(1-\prod_{i=1, j=i}^{n}\left(1-\left(1-\alpha_{i}^{2}\right)^{\xi}\left(1-\alpha_{j}^{2}\right)^{\zeta}\right)^{\frac{2}{n(n+1)}}\right)^{\frac{1}{\xi+\zeta}}}\right\}, \\
\left\{\left(\sqrt{1-\prod_{i=1, j=i}^{n}\left(1-\beta_{i}^{2 \xi} \beta_{j}^{2 \zeta}\right)^{\frac{2}{n(n+1)}}}\right)^{\frac{1}{\xi+\zeta}}\right\}
\end{array}\right\}
$$

Proof. Based on Definition 5:

$$
\begin{aligned}
& \xi \widetilde{p}_{i}=\cup_{\alpha_{i} \in h_{i}, \beta_{i} \in g_{i}}\left\{\left\{\sqrt{1-\left(1-\alpha_{i}^{2}\right)^{\xi}}\right\},\left\{\beta_{i}^{\xi}\right\}\right\} \\
& \zeta \widetilde{p}_{j}=\cup_{\alpha_{j} \in h_{j}, \beta_{j} \in g_{j}}\left\{\left\{\sqrt{1-\left(1-\alpha_{j}^{2}\right)^{\zeta}}\right\},\left\{\beta_{j}^{\zeta}\right\}\right\}
\end{aligned}
$$


Thus,

$$
\widetilde{\xi} \widetilde{p}_{i} \oplus \zeta \widetilde{p}_{j}=\cup_{\alpha_{i} \in h_{i}, \alpha_{j} \in h_{j}, \beta_{i} \in g_{i}, \alpha_{j} \in h_{j},}\left\{\left\{\sqrt{1-\left(1-\alpha_{i}^{2}\right)^{\xi}\left(1-\alpha_{j}^{2}\right)^{\zeta}}\right\},\left\{\beta_{i}^{\xi} \beta_{j}^{\zeta}\right\}\right\}
$$

Therefore,

$$
\begin{aligned}
& \bigotimes_{i=1}^{n} \otimes_{j=i}^{n}\left(\xi \widetilde{p}_{i} \oplus \widetilde{p_{j}}\right) \\
& =\cup_{\alpha_{i} \in h_{i}, \alpha_{j} \in h_{j}, \beta_{i} \in g_{i}, \alpha_{j} \in h_{j}}\left\{\begin{array}{l}
\left\{\sqrt{\prod_{i=1, j=i}^{n}\left(1-\left(1-\alpha_{i}^{2}\right)^{\xi}\left(1-\alpha_{j}^{2}\right)^{\zeta}\right)}\right\}, \\
\left\{\sqrt{1-\prod_{i=1, j=i}^{n}\left(1-\beta_{i}^{2 \xi} \beta_{j}^{2 \zeta}\right)}\right\}
\end{array}\right\}
\end{aligned}
$$

Furthermore,

$$
\begin{aligned}
& \left(\stackrel{\otimes}{i=1}_{j=i}^{n} \stackrel{\bigotimes}{n}_{j=i}\left(\xi \widetilde{p}_{i} \oplus \zeta \widetilde{p}_{j}\right)\right)^{\frac{2}{n(n+1)}} \\
& =\cup_{\alpha_{i} \in h_{i}, \alpha_{j} \in h_{j}, \beta_{i} \in g_{i}, \alpha_{j} \in h_{j}}\left\{\begin{array}{l}
\left\{\sqrt{\prod_{i=1, j=i}^{n}\left(1-\left(1-\alpha_{i}^{2}\right)^{\xi}\left(1-\alpha_{j}^{2}\right)^{\zeta}\right)^{\frac{2}{n(n+1)}}}\right\}, \\
\left\{\sqrt{1-\prod_{i=1, j=i}^{n}\left(1-\beta_{i}^{2 \xi} \beta_{j}^{2 \zeta}\right)^{\frac{2}{n(n+1)}}}\right\}
\end{array}\right\}
\end{aligned}
$$

Therefore,

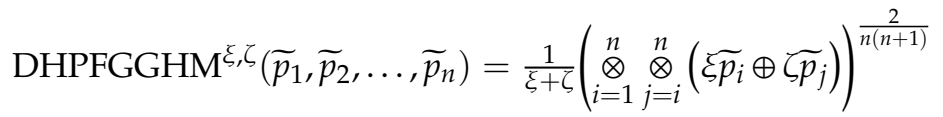

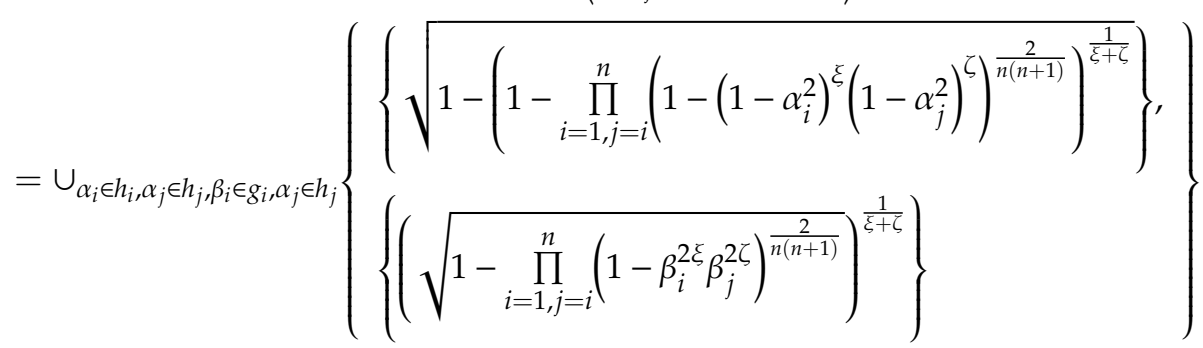

Thus, the proof have been finished.

Example 5. Assume that $\widetilde{p}_{1}=\{\{0.7,0.8\},\{0.4\}\}, \widetilde{p}_{2}=\{\{0.3\},\{0.6,0.7\}\}, \widetilde{p}_{3}=\{\{0.1,0.3\},\{0.4,0.6\}\}$ and $\widetilde{p}_{4}=\{\{0.5\},\{0.5\}\}$ are four DHPFNs, and suppose that $\xi=2, \zeta=3$. Then, according to the DHPFGGHM operator, we can obtain the fused results as follows. For the membership degree function $\alpha$, the fused results are shown as: 


$$
\begin{aligned}
& \alpha_{1}=\text { DHPFGGHM }{ }^{2,3}(0.7,0.3,0.1,0.5)=\sqrt{1-\left(1-\prod_{i=1, j=i}^{n}\left(1-\left(1-\alpha_{i}^{2}\right)^{\xi}\left(1-\alpha_{j}^{2}\right)^{\zeta}\right)^{\frac{2}{n(n+1)}}\right)^{\frac{1}{\xi+\zeta}}} \\
& =\sqrt{\left(\begin{array}{l}
\left(1-\left(1-0.7^{2}\right)^{2} \times\left(1-0.7^{2}\right)^{3}\right) \times\left(1-\left(1-0.7^{2}\right)^{2} \times\left(1-0.3^{2}\right)^{3}\right) \\
\times\left(1-\left(1-0.7^{2}\right)^{2} \times\left(1-0.1^{2}\right)^{3}\right) \times\left(1-\left(1-0.7^{2}\right)^{2} \times\left(1-0.5^{2}\right)^{3}\right) \\
1-\left(\begin{array}{l}
\left(1-\left(1-0.3^{2}\right)^{2} \times\left(1-0.3^{2}\right)^{3}\right) \times\left(1-\left(1-0.3^{2}\right)^{2} \times\left(1-0.1^{2}\right)^{3}\right) \\
\times\left(1-\left(1-0.3^{2}\right)^{2} \times\left(1-0.5^{2}\right)^{3}\right) \times\left(1-\left(1-0.1^{2}\right)^{2} \times\left(1-0.1^{2}\right)^{3}\right)
\end{array}\right) \\
\times\left(1-\left(1-0.1^{2}\right)^{2} \times\left(1-0.5^{2}\right)^{3}\right) \times\left(1-\left(1-0.5^{2}\right)^{2} \times\left(1-0.5^{2}\right)^{3}\right)
\end{array}\right)} \\
& = \\
& =0.3461
\end{aligned}
$$

Similarly, we can obtain

$$
\begin{aligned}
& \alpha_{2}=\operatorname{DHPFGGHM}^{2,3}(0.7,0.3,0.3,0.5)=0.4236 \\
& \alpha_{3}=\operatorname{DHPFGGHM}^{2,3}(0.8,0.3,0.1,0.5)=0.3545 \\
& \alpha_{4}=\operatorname{DHPFGGHM}^{2,3}(0.8,0.3,0.3,0.5)=0.4343
\end{aligned}
$$

Hence, we can get $\alpha=\{0.3461,0.4236,0.3545,0.4343\}$.

For the non-membership degree function $\beta$, the fused results are shown as:

$$
\begin{aligned}
& \beta_{1}=\text { DHPFGGHM }{ }^{2,3}(0.4,0.6,0.4,0.5)=\left(\sqrt{1-\prod_{i=1, j=i}^{n}\left(1-\beta_{i}^{2 \xi} \beta_{j}^{2 \zeta}\right)^{\frac{2}{n(n+1)}}}\right)^{\frac{1}{\xi+\zeta}} \\
& \left.=\left(\sqrt{1-\left(\begin{array}{l}
\left(1-0.4^{2 \times 2} \times 0.4^{2 \times 3}\right) \times\left(1-0.4^{2 \times 2} \times 0.6^{2 \times 3}\right) \times\left(1-0.4^{2 \times 2} \times 0.4^{2 \times 3}\right) \\
\times\left(1-0.4^{2 \times 2} \times 0.5^{2 \times 3}\right) \times\left(1-0.6^{2 \times 2} \times 0.6^{2 \times 3}\right) \times\left(1-0.6^{2 \times 2} \times 0.4^{2 \times 3}\right) \\
\times\left(1-0.6^{2 \times 2} \times 0.5^{2 \times 3}\right) \times\left(1-0.4^{2 \times 2} \times 0.4^{2 \times 3}\right) \times\left(1-0.4^{2 \times 2} \times 0.5^{2 \times 3}\right) \\
\times\left(1-0.5^{2 \times 2} \times 0.5^{2 \times 3}\right)
\end{array}\right.}\right)^{\frac{1}{10}}\right)^{\frac{1}{2+3}} \\
& =0.5100
\end{aligned}
$$

Similarly, we can obtain

$$
\begin{aligned}
& \beta_{2}=\operatorname{DHPFGGHM}^{2,3}(0.4,0.6,0.6,0.5)=0.5516 \\
& \beta_{3}=\operatorname{DHPFGGHM}^{2,3}(0.4,0.7,0.4,0.5)=0.5734 \\
& \beta_{4}=\operatorname{DHPFGGHM}^{2,3}(0.4,0.7,0.6,0.5)=0.5968
\end{aligned}
$$

Hence, we can get $\beta=\{0.5100,0.5516,0.5734,0.5968\}$.Therefore,

$$
\operatorname{DHPFGGHM}\left(\widetilde{p}_{1}, \widetilde{p}_{2}, \widetilde{p}_{3}, \widetilde{p}_{4}\right)=\left\{\begin{array}{c}
\{0.3461,0.4236,0.3545,0.4343\} \\
\{0.5100,0.5516,0.5734,0.5968\}
\end{array}\right\} .
$$


It can be easily proven that the DHPFGGHM operator satisfies the following properties.

Property 7. (Idempotency) If all $\widetilde{p}_{j}=\left(h_{j}, g_{j}\right)(j=1,2, \cdots, n)$ are equal, i.e., $\tilde{p}_{j}=\tilde{p}$ for all $j$, then

$$
\operatorname{DHPFGGHM}^{\xi, \zeta}\left(\widetilde{p}_{1}, \widetilde{p}_{2}, \ldots, \widetilde{p}_{n}\right)=\tilde{p}
$$

Property 8. (Boundedness) Let $\widetilde{p}_{j}=\left(h_{j}, g_{j}\right)(j=1,2, \cdots, n)$ be a collection of DHPFNs, and let

$$
\widetilde{p}^{+}=\cup_{\alpha_{j} \in h_{j}, \beta_{j} \in g_{j}}\left\{\left\{\max _{i}\left(\alpha_{i}\right)\right\},\left\{\min _{i}\left(\beta_{i}\right)\right\}\right\}, \widetilde{p}^{-}=\cup_{\alpha_{j} \in h_{j}, \beta_{j} \in g_{j}}\left\{\left\{\min _{i}\left(\alpha_{i}\right)\right\},\left\{\max _{i}\left(\beta_{i}\right)\right\}\right\}
$$

Then

$$
\widetilde{p}^{-} \leq \operatorname{DHPFGGHM}{ }^{\xi, \zeta}\left(\widetilde{p}_{1}, \widetilde{p}_{2}, \ldots, \widetilde{p}_{n}\right) \leq \widetilde{p}^{+}
$$

Property 9. (Monotonicity) Let $\widetilde{p}_{j}=\left(h_{j}, g_{j}\right)$ and $\widetilde{p}_{j}^{\prime}=\left(h_{j}^{\prime}, g_{j}^{\prime}\right), j=1,2, \cdots, n$, be two set of DHPFNs. If $\widetilde{p}_{j} \leq \widetilde{p}_{j}^{\prime}$, for all $j$, then

$$
\operatorname{DHPFGGHM}^{\xi, \zeta}\left(\widetilde{p}_{1}, \widetilde{p}_{2}, \ldots, \widetilde{p}_{n}\right) \leq \operatorname{DHPFGGHM}^{\xi, \zeta}\left(\vec{p}_{1}, \widetilde{p}_{2}, \ldots, \vec{p}_{n}\right)
$$

\subsection{The DHPFGGWHM Aggregation Operator}

Using Definition 11, we can conclude that the DHPFGGHM operator didn't take the importance of arguments being fused into account. However, in many practical MADM problems, we should consider the weights of attributes. To overcome the limitations of the DHPFGGHM operator, we propose a novel DHPFGGWHM operator as follows.

Definition 12. Assume that $\xi, \zeta>0$ and $\widetilde{p}_{j}=\left(h_{j}, g_{j}\right)(j=1,2, \cdots, n)$ are a collection of DHPFNs. Then, the DHPFGGWHM operator can be defined as:

$$
\operatorname{DHPFGGWHM}_{w}^{\xi, \zeta}\left(\widetilde{p}_{1}, \widetilde{p}_{2}, \ldots, \widetilde{p}_{n}\right)=\frac{1}{\xi+\zeta}\left(\stackrel{\otimes}{i=1}_{j=i}^{n} \stackrel{\otimes}{\otimes}_{j=i}\left(\xi \widetilde{p}_{i} \oplus \zeta \widetilde{p}_{j}\right)^{w_{i} w_{j}}\right)
$$

According to the operation laws of the DHPFNs described in Definition 5, we can obtain Theorem 4.

Theorem 4. Assume that $\xi, \zeta>0$ and $\widetilde{p}_{j}=\left(h_{j}, g_{j}\right)(j=1,2, \cdots, n)$ are a collection of DHPFNs with the weighting vector $w=\left(w_{1}, w_{2}, \ldots, w_{n}\right)^{T}$ which satisfies $w_{j}>0, i=1,2, \ldots, n$ and $\sum_{j=1}^{n} w_{j}=1$. Then, their fused result obtained by utilizing the DHPFGGWHM operator is also a DHPFN, and

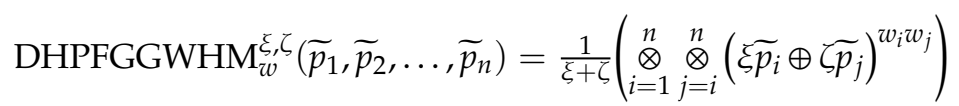

$$
\begin{aligned}
& =\cup_{\alpha_{i} \in h_{i}, \alpha_{j} \in h_{j}, \beta_{i} \in g_{i}, \alpha_{j} \in h_{j},}\left\{\begin{array}{l}
\left\{\sqrt{1-\left(1-\prod_{i=1, j=i}^{n}\left(1-\left(1-\alpha_{i}^{2}\right)^{\xi}\left(1-\alpha_{j}^{2}\right)^{\zeta}\right)^{w_{i} w_{j}}\right)^{\frac{1}{\xi+\zeta}}}\right\}, \\
\left\{\left(\sqrt{1-\prod_{i=1, j=i}^{n}\left(1-\beta_{i}^{2 \xi} \beta_{j}^{2 \zeta}\right)^{w_{i} w_{j}}}\right)^{\frac{1}{\xi+\zeta}}\right\}
\end{array}\right\}
\end{aligned}
$$


Proof. Based on Definition 5, we can obtain:

$$
\begin{gathered}
\xi \widetilde{p}_{i}=\cup_{\alpha_{i} \in h_{i}, \beta_{i} \in g_{i}}\left\{\left\{\sqrt{1-\left(1-\alpha_{i}^{2}\right)^{\xi}}\right\},\left\{\beta_{i}^{\xi}\right\}\right\} \\
\widetilde{\zeta p}_{j}=\cup_{\alpha_{j} \in h_{j}, \beta_{j} \in g_{j}}\left\{\left\{\sqrt{1-\left(1-\alpha_{j}^{2}\right)^{\zeta}}\right\},\left\{\beta_{j}^{\zeta}\right\}\right\}
\end{gathered}
$$

Thus,

$$
\xi \widetilde{p}_{i} \oplus \zeta \widetilde{p}_{j}=\cup_{\alpha_{i} \in h_{i}, \alpha_{j} \in h_{j}, \beta_{i} \in g_{i}, \alpha_{j} \in h_{j},}\left\{\left\{\sqrt{1-\left(1-\alpha_{i}^{2}\right)^{\xi}\left(1-\alpha_{j}^{2}\right)^{\zeta}}\right\},\left\{\beta_{i}^{\xi} \beta_{j}^{\zeta}\right\}\right\}
$$

Therefore,

$$
\begin{aligned}
& \left(\xi \widetilde{p}_{i} \oplus \zeta \widetilde{p}_{j}\right)^{w_{i} w_{j}}
\end{aligned}
$$

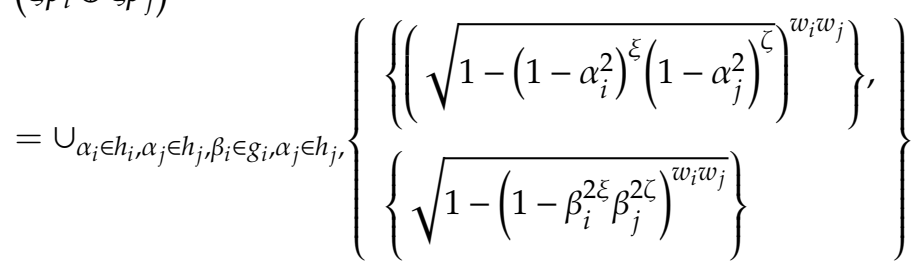

Thereafter,

$$
\begin{aligned}
& \bigotimes_{i=1}^{n} \otimes_{j=i}^{n}\left(\xi \widetilde{p}_{i} \oplus \zeta \widetilde{p}_{j}\right)^{w_{i} w_{j}} \\
& =\cup_{\alpha_{i} \in h_{i}, \alpha_{j} \in h_{j}, \beta_{i} \in g_{i}, \alpha_{j} \in h_{j},}\left\{\begin{array}{l}
\left\{\left(\sqrt{\prod_{i=1, j=i}^{n}\left(1-\left(1-\alpha_{i}^{2}\right)^{\xi}\left(1-\alpha_{j}^{2}\right)^{\zeta}\right)}\right)^{w_{i} w_{j}}\right\}, \\
\left\{\sqrt{1-\prod_{i=1, j=i}^{n}\left(1-\beta_{i}^{2 \xi} \beta_{j}^{2 \zeta}\right)^{w_{i} w_{j}}}\right\}
\end{array}\right\}
\end{aligned}
$$

Therefore,

$$
\begin{aligned}
& \operatorname{DHPFGGWHM}_{w}^{\xi, \zeta}\left(\widetilde{p}_{1}, \widetilde{p}_{2}, \ldots, \widetilde{p}_{n}\right)=\frac{1}{\xi+\zeta}\left(\underset{i=1}{\otimes} \underset{j=i}{\otimes}\left(\xi \widetilde{p}_{i} \oplus \zeta \widetilde{p}_{j}\right)^{w_{i} w_{j}}\right) \\
& =\cup_{\alpha_{i} \in h_{i}, \alpha_{j} \in h_{j}, \beta_{i} \in g_{i}, \alpha_{j} \in h_{j},}\left\{\begin{array}{l}
\left\{\sqrt{1-\left(1-\prod_{i=1, j=i}^{n}\left(1-\left(1-\alpha_{i}^{2}\right)^{\xi}\left(1-\alpha_{j}^{2}\right)^{\zeta}\right)^{w_{i} w_{j}}\right)^{\frac{1}{\xi+\zeta}}}\right\}, \\
\left\{\left(\sqrt{1-\prod_{i=1, j=i}^{n}\left(1-\beta_{i}^{2 \xi} \beta_{j}^{2 \zeta}\right)^{w_{i} w_{j}}}\right)^{\frac{1}{\xi+\zeta}}\right\}
\end{array}\right\}
\end{aligned}
$$

Thus, we have finished the proof.

Example 6. Assume that $\widetilde{p}_{1}=\{\{0.7,0.8\},\{0.4\}\}, \widetilde{p}_{2}=\{\{0.3\},\{0.6,0.7\}\}, \widetilde{p}_{3}=\{\{0.1,0.3\},\{0.4,0.6\}\}$ and $\tilde{p}_{4}=\{\{0.5\},\{0.5\}\}$ are four DHPFNs, and suppose that $\xi=2, \zeta=3$ and $w_{j}=(0.3,0.2,0.1,0.4)$. Then, according to the DHPFGGWHM operator, we can obtain the fused results as follows. For the membership degree function $\alpha$, the fused results are shown as: 


$$
\begin{aligned}
& \alpha_{1}=\operatorname{DHPFGGWHM}{ }^{2,3}(0.7,0.3,0.1,0.5)=\sqrt{1-\left(1-\prod_{i=1, j=i}^{n}\left(1-\left(1-\alpha_{i}^{2}\right)^{\xi}\left(1-\alpha_{j}^{2}\right)^{\zeta}\right)^{w_{i} w_{j}}\right)^{\frac{1}{\xi+\zeta}}} \\
& =\sqrt{\left(\begin{array}{l}
1-\left(1-\left(1-0.7^{2}\right)^{2} \times\left(1-0.7^{2}\right)^{3}\right)^{0.3 \times 0.3} \times\left(1-\left(1-0.7^{2}\right)^{2} \times\left(1-0.3^{2}\right)^{3}\right)^{0.3 \times 0.2} \\
\times\left(1-\left(1-0.7^{2}\right)^{2} \times\left(1-0.1^{2}\right)^{3}\right)^{0.3 \times 0.1} \times\left(1-\left(1-0.7^{2}\right)^{2} \times\left(1-0.5^{2}\right)^{3}\right)^{0.3 \times 0.4} \\
\times\left(1-\left(1-0.3^{2}\right)^{2} \times\left(1-0.3^{2}\right)^{3}\right)^{0.2 \times 0.2} \times\left(1-\left(1-0.3^{2}\right)^{2} \times\left(1-0.1^{2}\right)^{3}\right)^{0.2 \times 0.1} \\
\times\left(1-\left(1-0.3^{2}\right)^{2} \times\left(1-0.5^{2}\right)^{3}\right)^{0.2 \times 0.4} \times\left(1-\left(1-0.1^{2}\right)^{2} \times\left(1-0.1^{2}\right)^{3}\right)^{0.1 \times 0.1} \\
\times\left(1-\left(1-0.1^{2}\right)^{2} \times\left(1-0.5^{2}\right)^{3}\right)^{0.1 \times 0.4} \times\left(1-\left(1-0.5^{2}\right)^{2} \times\left(1-0.5^{2}\right)^{3}\right)^{0.4 \times 0.4}
\end{array}\right.} \\
& =0.5156
\end{aligned}
$$

Similarly, we can obtain

$$
\begin{aligned}
& \alpha_{2}=\operatorname{DHPFGGWHM}^{2,3}(0.7,0.3,0.3,0.5)=0.5378 \\
& \alpha_{3}=\operatorname{DHPFGGWHM}^{2,3}(0.8,0.3,0.1,0.5)=0.5273 \\
& \alpha_{4}=\text { DHPFGGWHM }^{2,3}(0.8,0.3,0.3,0.5)=0.5503
\end{aligned}
$$

Hence, we can get $\alpha=\{0.5156,0.5378,0.5273,0.5503\}$.

For the non-membership degree function $\beta$, the fused results are shown as:

$\beta_{1}=\operatorname{DHPFGGWHM}^{2,3}(0.4,0.6,0.4,0.5)=\left(\sqrt{1-\prod_{i=1, j=i}^{n}\left(1-\beta_{i}^{2 \xi} \beta_{j}^{2 \zeta}\right)^{w_{i} w_{j}}}\right)^{\frac{1}{\xi+\zeta}}$

$=\left(\begin{array}{l}\begin{array}{l}1-\left(1-0.4^{2 \times 2} \times 0.4^{2 \times 3}\right)^{0.3 \times 0.3} \times\left(1-0.4^{2 \times 2} \times 0.6^{2 \times 3}\right)^{0.3 \times 0.2} \times\left(1-0.4^{2 \times 2} \times 0.4^{2 \times 3}\right)^{0.3 \times 0.1} \\ \times\left(1-0.4^{2 \times 2} \times 0.5^{2 \times 3}\right)^{0.3 \times 0.4} \times\left(1-0.6^{2 \times 2} \times 0.6^{2 \times 3}\right)^{0.2 \times 0.2} \times\left(1-0.6^{2 \times 2} \times 0.4^{2 \times 3}\right)^{0.2 \times 0.1} \\ \times\left(1-0.6^{2 \times 2} \times 0.5^{2 \times 3}\right)^{0.2 \times 0.4} \times\left(1-0.4^{2 \times 2} \times 0.4^{2 \times 3}\right)^{0.1 \times 0.1} \times\left(1-0.4^{2 \times 2} \times 0.5^{2 \times 3}\right)^{0.1 \times 0.4} \\ \times\left(1-0.5^{2 \times 2} \times 0.5^{2 \times 3}\right)^{0.4 \times 0.4}\end{array}\end{array}\right.$

$=0.4850$

Similarly, we can obtain

$$
\begin{aligned}
& \beta_{2}=\operatorname{DHPFGGWHM}^{2,3}(0.4,0.6,0.6,0.5)=0.5006 \\
& \beta_{3}=\operatorname{DHPFGGWHM}^{2,3}(0.4,0.7,0.4,0.5)=0.5338 \\
& \beta_{4}=\operatorname{DHPFGGWHM}^{2,3}(0.4,0.7,0.6,0.5)=0.5433
\end{aligned}
$$

Hence, we can get $\beta=\{0.4850,0.5006,0.5338,0.5433\}$. Therefore,

$$
\operatorname{DHPFGGWHM}\left(\widetilde{p}_{1}, \widetilde{p}_{2}, \widetilde{p}_{3}, \widetilde{p}_{4}\right)=\left\{\begin{array}{l}
\{0.5156,0.5378,0.5273,0.5503\}, \\
\{0.4850,0.5006,0.5338,0.5433\}
\end{array}\right\} .
$$


It can be easily proven that the DHPFGGWHM operator satisfies the following properties.

Property 10. (Idempotency) If all $\tilde{p}_{j}=\left(h_{j}, g_{j}\right)(j=1,2, \cdots, n)$ are equal, i.e., $\tilde{p}_{j}=\tilde{p}$ for all $j$, then

$$
\operatorname{DHPFGGWHM}{ }_{w}^{\xi, \zeta}\left(\widetilde{p}_{1}, \widetilde{p}_{2}, \ldots, \widetilde{p}_{n}\right)=\widetilde{p}
$$

Property 11. (Boundedness) Let $\widetilde{p}_{j}=\left(h_{j}, g_{j}\right)(j=1,2, \cdots, n)$ be a collection of DHPFNs, and let

$$
\widetilde{p}^{+}=\cup_{\alpha_{j} \in h_{j}, \beta_{j} \in g_{j}}\left\{\left\{\max _{i}\left(\alpha_{i}\right)\right\},\left\{\min _{i}\left(\beta_{i}\right)\right\}\right\}, \widetilde{p}^{-}=\cup_{\alpha_{j} \in h_{j}, \beta_{j} \in g_{j}}\left\{\left\{\min _{i}\left(\alpha_{i}\right)\right\},\left\{\max _{i}\left(\beta_{i}\right)\right\}\right\}
$$

Then

$$
\widetilde{p}^{-} \leq \operatorname{DHPFGGWHM} \mathcal{w}_{w}^{\xi, \zeta}\left(\widetilde{p}_{1}, \widetilde{p}_{2}, \ldots, \widetilde{p}_{n}\right) \leq \widetilde{p}^{+}
$$

Property 12. (Monotonicity) Let $\widetilde{p}_{j}=\left(h_{j}, g_{j}\right)$ and $\widetilde{p}_{j}^{\prime}=\left(h_{j}^{\prime}, g_{j}^{\prime}\right), j=1,2, \cdots, n$, be two sets of DHPFNs. If $\widetilde{p}_{j} \leq \widetilde{p}_{j}^{\prime}$ for all $j$, then

$$
\operatorname{DHPFGGWHM}_{w}^{\xi, \zeta}\left(\widetilde{p}_{1}, \widetilde{p}_{2}, \ldots, \widetilde{p}_{n}\right) \leq \operatorname{DHPFGGWHM}_{w}^{\xi, \zeta}\left(\widetilde{p}_{1}, \vec{p}_{2}, \ldots, \vec{p}_{n}\right)
$$

\section{An Approach to MADM with DHPFNs Information}

In this section, we shall use the DHPFGWHM and DHPFGGWHM operators to deal with MADM problems with dual hesitant Pythagorean fuzzy information. Suppose that there are $m$ alternatives $\eta=\left\{\eta_{1}, \eta_{2}, \cdots, \eta_{m}\right\}$, and each alternative is characterized by $n$ attributes $\delta=\left\{\delta_{1}, \delta_{2}, \cdots, \delta_{n}\right\}$ with the weighting vector being $w_{j}=\left\{w_{1}, w_{2}, \cdots, w_{n}\right\}$. Then, the dual hesitant Pythagorean fuzzy matrix can be constructed as $\widetilde{P}=\left(\widetilde{p}_{i j}\right)_{m \times n^{\prime}}$, with each element $\widetilde{p}_{i j}=\left(h_{i j}, g_{i j}\right)(i=1,2, \cdots, m, j=1,2, \cdots, n)$ indicating a dual hesitant Pythagorean fuzzy number, where $h_{i j}$ means the membership degree set with several values in $[0,1]$, and $g_{i j}$ means the no-membership degree set with several values in $[0,1]$.

In what follows, we apply the DHPFGWHM or DHPFGGWHM operator to MADM problems for supplier selection in supply chain management with dual hesitant Pythagorean fuzzy information.

Step 1. In order to derive the fused results of each alternative, for alternatives $\eta=\left\{\eta_{1}, \eta_{2}, \cdots, \eta_{m}\right\}$, based on the weighting vector $w_{j}=\left\{w_{1}, w_{2}, \cdots, w_{n}\right\}$ and dual hesitant Pythagorean fuzzy information $\widetilde{p}_{i j}=\left(h_{i j}, g_{i j}\right)(i=1,2, \cdots, m, j=1,2, \cdots, n)$ given in matrix $\widetilde{P}=\left(\widetilde{p}_{i j}\right)_{m \times n^{\prime}}$, we can aggregate all the DHPFNs by the DHPFGWHM operator

$$
\begin{aligned}
& \widetilde{p}_{i}=\operatorname{DHPFGWHM} M_{w}^{\xi, \zeta}\left(\widetilde{p}_{i 1}, \widetilde{p}_{i 2}, \ldots, \widetilde{p}_{i n}\right)=\left({\underset{⿴ 囗 十}{n}}_{k=1}^{n} \bigoplus_{j=k}^{n}\left(w_{i k} w_{i j}\left(\widetilde{p}_{i k}^{\xi} \widetilde{p}_{i j}^{\zeta}\right)\right)\right)^{\frac{1}{\xi+\zeta}} \\
& =\cup_{\alpha_{i k} \in h_{i k}, \alpha_{i j} \in h_{i j}, \beta_{i j} \in g_{i j}, \alpha_{i j} \in h_{i j},}\left\{\begin{array}{l}
\left\{\left(\sqrt{1-\prod_{k=1, j=k}^{n}\left(1-\alpha_{i k}^{2 \xi} \alpha_{i j}^{2 \zeta}\right)^{w_{i} w_{j}}}\right)^{\frac{1}{\xi+\zeta}}\right\}, \\
\left\{\left(\sqrt{1-\left(1-\prod_{k=1, j=k}^{n}\left(1-\left(1-\beta_{i k}^{2}\right)^{\xi}\left(1-\beta_{i j}^{2}\right)^{\zeta}\right)^{w_{i} w_{j}}\right)^{\frac{1}{\xi+\zeta}}}\right)\right\}
\end{array}\right\}
\end{aligned}
$$


or the DHPFGGWHM operator

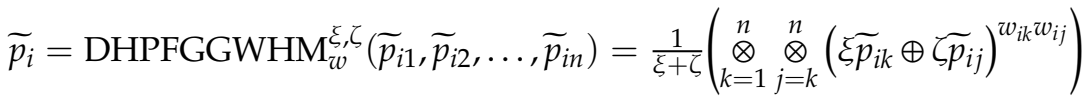

$$
\begin{aligned}
& =\cup_{\alpha_{i k} \in h_{i k}, \alpha_{i j} \in h_{i j}, \beta_{i j} \in g_{i j}, \alpha_{i j} \in h_{i j},}\left\{\begin{array}{l}
\left\{\left(\sqrt{1-\left(1-\prod_{k=1, j=k}^{n}\left(1-\left(1-\alpha_{i k}^{2}\right)^{\xi}\left(1-\alpha_{i j}^{2}\right)^{\zeta}\right)^{w_{i} w_{j}}\right)^{\frac{1}{\xi+\zeta}}}\right)\right\}, \\
\left\{\left(\sqrt{1-\prod_{k=1, j=k}^{n}\left(1-\beta_{i k}^{2 \xi} \beta_{i j}^{2 \zeta}\right)^{w_{i} w_{j}}}\right)^{\frac{1}{\xi+\zeta}}\right\}
\end{array}\right\}
\end{aligned}
$$

to obtain the overall fused results $\widetilde{p}_{i}(i=1,2, \cdots, m)$.

Step 2. To obtain the rank of all the alternatives, we need to adapt the score function and accuracy function described in Definition 4. Firstly, based on the score function equation, we can compute the score values $S\left(\widetilde{p}_{i}\right)(i=1,2, \cdots, m)$ of $\widetilde{p}_{i}(i=1,2, \cdots, m)$. If all the score values of $\widetilde{p}_{i}(i=1,2, \cdots, m)$ are different, we can easily obtain the ordering of alternatives. Then, if there is no difference between any two scores $S\left(\widetilde{p}_{i}\right)$ and $S\left(\widetilde{p}_{j}\right)$, we need to compute the accuracy values $H\left(\widetilde{p}_{i}\right)$ and $H\left(\widetilde{p}_{j}\right)$ of $\widetilde{p}_{i}$ and $\widetilde{p}_{j}$, respectively, and then determine the ordering of all the alternatives $\eta_{i}$ and $\eta_{j}$ based on the accuracy results $H\left(\widetilde{p}_{i}\right)$ and $H\left(\widetilde{p}_{j}\right)$.

Step 3. Determine the ordering of all the alternatives $\eta_{i}(i=1,2, \cdots, m)$ and select the best one(s) according to the scores values $S\left(\widetilde{p}_{i}\right)(i=1,2, \cdots, m)$ and accuracy results $H\left(\widetilde{p}_{i}\right)$. Thus, we have finished the decision making process by using the DHPFGWHM operator or the DHPFGGWHM operator.

\section{Numerical Example and Comparative Analysis}

\subsection{Numerical Example}

In this section we present a numerical example for supplier selection in supply chain management with dual hesitant Pythagorean fuzzy information in order to demonstrate the method proposed in this paper. Suppose there is a problem to do with the supplier selection in supply chain management which is a classical MADM problem. There are five prospective suppliers $\eta_{i}(i=1,2,3,4,5)$ for four attributes $\delta_{j}(j=1,2,3,4)$. The four attributes include product quality $\left(\delta_{1}\right)$, service $\left(\delta_{2}\right)$, delivery, $\left(\delta_{3}\right)$ and price $\left(\delta_{4}\right)$, respectively. In order to avoid influencing each other, the decision makers are required to evaluate the five suppliers $\eta_{i}(i=1,2,3,4,5)$ under the above four attributes in anonymity. The decision matrix $\widetilde{P}=\left(\widetilde{p}_{i j}\right)_{5 \times 4}$ is presented in Table 1 , where $\widetilde{p}_{i j}(i=1,2,3,4,5, j=1,2,3,4)$ are in the form of DHPFNs. (Suppose the weighting vector is $w_{j}=(0.25,0.34,0.27,0.14)$ )

Table 1. Dual hesitant Pythagorean fuzzy decision matrix.

\begin{tabular}{ccccc}
\hline Alternatives & $\delta_{\mathbf{1}}$ & $\delta_{\mathbf{2}}$ & $\delta_{\mathbf{3}}$ & $\boldsymbol{\delta}_{\mathbf{4}}$ \\
\hline$\eta_{1}$ & $\{\{0.4,0.5\},\{0.7\}\}$ & $\{\{0.5,0.6\},\{0.4,0.5)\}$ & $\{\{0.3,0.4\},\{0.8)\}$ & $\{\{0.5,0.6\},\{0.6\}\}$ \\
$\eta_{2}$ & $\{\{0.7\},\{0.5\}\}$ & $\{\{0.3,0.5,0.6\},\{0.5\}\}$ & $\{\{0.3\},\{0.7,0.8,0.9\}\}$ & $\{\{0.6),\{0.5,0.6)\}$ \\
$\eta_{3}$ & $\{\{0.6,0.8\},\{0.3\}\}$ & $\{\{0.3\},\{0.8,0.9\}\}$ & $\{\{0.3,0.4,0.5\},\{0.7\}\}$ & $\{\{0.6,0.7,0.8\},\{0.4\}\}$ \\
$\eta_{4}$ & $\{\{0.8\},\{0.4\})\}$ & $\{\{0.7,0.8,0.9\},\{0.3\}\}$ & $\{\{0.2,0.3\},\{0.4\}\}$ & $\{\{0.2\},\{0.7,0.8,0.9\}\}$ \\
$\eta_{5}$ & $\{\{0.1,0.2\},\{0.3\}\}$ & $\{\{0.3,0.4,0.5\},\{0.6\}\}$ & $\{\{0.5,0.6\},\{0.3\}\}$ & $\{\{0.3,0.4,0.5\},\{0.6\}\}$ \\
\hline
\end{tabular}

In what follows, we can utilize our developed methods to deal with the supplier selection in supply chain management with dual hesitant Pythagorean fuzzy information.

Step 1. We aggregate the dual hesitant Pythagorean fuzzy information given in the matrix by utilizing the DHPFGWHM operator to obtain the overall preference values $\widetilde{p}_{i}$ of the supplier in supply chain 
management $\eta_{i}(i=1,2,3,4,5)$. Taking the alternative $\eta_{1}$ as an example (here, we take $\xi=\zeta=2$ ), we have

$$
\begin{aligned}
& \widetilde{p}_{1}=\operatorname{DHPFGWHM} M_{w}^{\xi, \zeta}\left(\widetilde{p}_{11}, \widetilde{p}_{12}, \widetilde{p}_{13}, \widetilde{p}_{14}\right)=\left(\underset{k=1}{\stackrel{4}{\oplus}} \underset{j=k}{\oplus}\left(w_{i k} w_{i j}\left(\widetilde{p}_{i k}^{\zeta} \widetilde{p}_{i j}^{\zeta}\right)\right)\right)^{\frac{1}{\xi+\zeta}}
\end{aligned}
$$

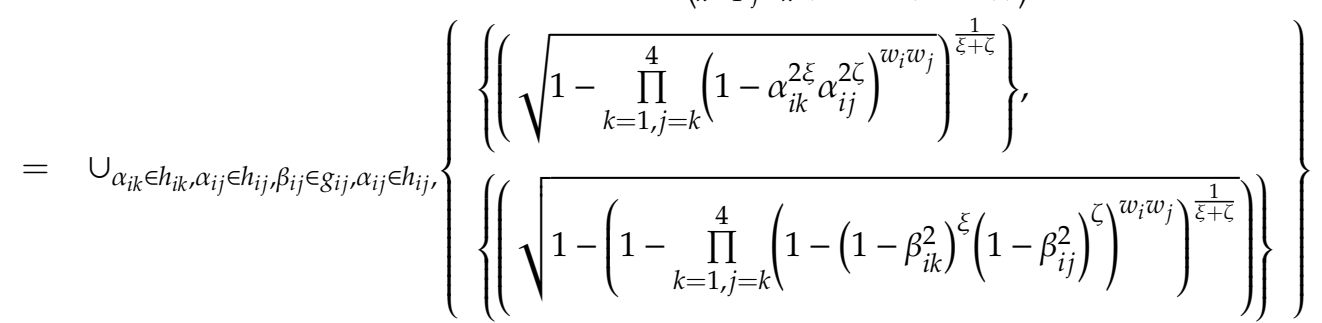

$$
\begin{aligned}
& =\{\{\{0.4,0.5\},\{0.7\}\},\{\{0.5,0.6\},\{0.4,0.5)\},\{\{0.3,0.4\},\{0.8)\},\{\{0.5,0.6\},\{0.6\}\}\} \\
& =\{\{0.4234,0.4461,0.4335,0.4547,0.4824,0.4964,0.4887,0.5023,0.4448,0.4642 \text {, } \\
& 0.4536,0.4719,0.4957,0.5087,0.5016,0.5143\},\{0.6319,0.6725\}\}
\end{aligned}
$$

Step 2. Compute the scores results $s\left(\widetilde{p}_{i}\right)(i=1,2,3,4,5)$ of the overall dual hesitant Pythagorean fuzzy preference values $\widetilde{p}_{i}(i=1,2,3,4,5)$ :

$$
\begin{aligned}
& s\left(\widetilde{p}_{1}\right)=0.3998, s\left(\widetilde{p}_{2}\right)=0.4536, s\left(\widetilde{p}_{3}\right)=0.4669 \\
& s\left(\widetilde{p}_{4}\right)=0.6255, s\left(\widetilde{p}_{5}\right)=0.4674
\end{aligned}
$$

Step 3. Determine the ordering of all the suppliers $\eta_{i}(i=1,2,3,4,5)$ based on the scores values $s\left(\widetilde{p}_{i}\right)(i=1,2,3,4,5): \eta_{4}>\eta_{5}>\eta_{3}>\eta_{2}>\eta_{1}$, and it is clear that the most desirable supplier is $\eta_{4}$.

Similarly, if we utilize the DHPFGGWHM operator to solve this MADM, the decision making steps can be described as follows.

Step 1'. Aggregate all dual hesitant Pythagorean fuzzy values $\widetilde{p}_{i j}(j=1,2,3,4)$ by using the DHPFGGWHM operator to derive the overall dual hesitant Pythagorean fuzzy values $\widetilde{p}_{i}(i=1,2, \cdots, 5)$ of the supplier $\eta_{i}$. Taking supplier $\eta_{1}$ for an example (here, we take $\xi=\zeta=2$ ), we have

$$
\begin{aligned}
& \widetilde{p}_{1}=\operatorname{DHPFGGWHM} \mathrm{w}_{w}^{\xi, \zeta}\left(\widetilde{p}_{11}, \widetilde{p}_{12}, \widetilde{p}_{13}, \widetilde{p}_{14}\right)=\frac{1}{\xi+\zeta}\left(\underset{k=1}{\otimes} \underset{j=k}{\otimes} \underset{\otimes}{\otimes}\left(\xi \widetilde{p}_{i k} \oplus \zeta \widetilde{p}_{i j}\right)^{w_{i k} w_{i j}}\right) \\
& =\cup_{\alpha_{i k} \in h_{i k}, \alpha_{i j} \in h_{i j}, \beta_{i j} \in \alpha_{i j}, \alpha_{i j} \in h_{i j},}\left\{\begin{array}{l}
\left\{\left(\sqrt{1-\left(1-\prod_{k=1, j=k}^{4}\left(1-\left(1-\alpha_{i k}^{2}\right)^{\xi}\left(1-\alpha_{i j}^{2}\right)^{\zeta}\right)^{w_{i} w_{j}}\right)^{\frac{1}{\xi+\zeta}}}\right)\right\}, \\
\left\{\left(\sqrt{1-\prod_{k=1, j=k}^{4}\left(1-\beta_{i k}^{2 \xi} \beta_{i j}^{2 \zeta}\right)^{w_{i} w_{j}}}\right)^{\frac{1}{\xi+\zeta}}\right\}
\end{array}\right\} \\
& =\{\{\{0.4,0.5\},\{0.7\}\},\{\{0.5,0.6\},\{0.4,0.5)\},\{\{0.3,0.4\},\{0.8)\},\{\{0.5,0.6\},\{0.6\}\}\} \\
& =\{\{0.4929,0.5022,0.5190,0.5287,0.5186,0.5283,0.5460,0.5560,0.5140,0.5236 \text {, } \\
& 0.5406,0.5506,0.5406,0.5505,0.5686,0.5790\},\{0.6421,0.6493\}\}
\end{aligned}
$$

Step $2^{\prime}$. Compute the scores results $s\left(\widetilde{p}_{i}\right)(i=1,2,3,4,5)$ of the overall dual hesitant Pythagorean fuzzy values $\widetilde{p}_{i}(i=1,2,3,4,5)$ of the supplier $\widetilde{p}_{i}$ :

$$
\begin{aligned}
& s\left(\widetilde{p}_{1}\right)=0.4349, s\left(\widetilde{p}_{2}\right)=0.4549, s\left(\widetilde{p}_{3}\right)=0.3976 \\
& s\left(\widetilde{p}_{4}\right)=0.5240, s\left(\widetilde{p}_{5}\right)=0.4780
\end{aligned}
$$


Step $3^{\prime}$. Determine the ordering of all the suppliers $\eta_{i}(i=1,2,3,4,5)$ based on the score results $s\left(\widetilde{p}_{i}\right)(i=1,2,3,4,5)$ of $\widetilde{p}_{i}(i=1,2, \cdots, 5): \eta_{4}>\eta_{5}>\eta_{2}>\eta_{1}>\eta_{3}$ and it is clear that the most desirable supplier in supply chain management is $\eta_{4}$.

According to the above analysis, we can easily find that although the overall rating values of the alternatives are slightly different by using two operators respectively, the most desirable supplier in supply chain management is $\eta_{4}$.

\subsection{Influence of Parameters on the Final Result}

The parameters $\xi$ and $\zeta$ play an important role in the final ranking of alternatives. We may obtain different ordering results by assigning different values to $\xi$ and $\zeta$. By altering the values of $\xi$ and $\zeta$, different ranking results are obtained, as shown in Tables 2 and 3 . Therefore, the DHPFGWHM and DHPFGGWHM operators are shown to be considerably flexible by using a parameter vector. Tables 2 and 3 show that the ranking results increase and become steady with the increase of values in the parameter vector. That is, the final results become increasingly objective by considering the interrelationship among the attribute values. These features of the DHPFGWHM and DHPFGGWHM operators are crucial in real MADM problems.

Table 2. Ordering by the DHPFGWHM operators.

\begin{tabular}{ccccccc}
\hline Parameter & $s\left(\eta_{\mathbf{1}}\right)$ & $s\left(\eta_{\mathbf{2}}\right)$ & $s\left(\eta_{\mathbf{3}}\right)$ & $s\left(\eta_{\mathbf{4}}\right)$ & $s\left(\eta_{5}\right)$ & Ordering \\
\hline$\xi=\zeta=0.5$ & 0.2989 & 0.3322 & 0.3126 & 0.4804 & 0.3732 & $\eta_{4}>\eta_{5}>\eta_{2}>\eta_{3}>\eta_{1}$ \\
$\xi=\zeta=1$ & 0.3504 & 0.3921 & 0.3834 & 0.5540 & 0.4208 & $\eta_{4}>\eta_{5}>\eta_{2}>\eta_{3}>\eta_{1}$ \\
$\xi=\zeta=2$ & 0.3998 & 0.4536 & 0.4669 & 0.6255 & 0.4674 & $\eta_{4}>\eta_{5}>\eta_{3}>\eta_{2}>\eta_{1}$ \\
$\xi=\zeta=3$ & 0.4288 & 0.4887 & 0.5197 & 0.6621 & 0.4933 & $\eta_{4}>\eta_{3}>\eta_{5}>\eta_{2}>\eta_{1}$ \\
$\xi=\zeta=4$ & 0.4493 & 0.5117 & 0.5559 & 0.6846 & 0.5108 & $\eta_{4}>\eta_{3}>\eta_{2}>\eta_{5}>\eta_{1}$ \\
$\xi=\zeta=5$ & 0.4647 & 0.5280 & 0.5822 & 0.7001 & 0.5235 & $\eta_{4}>\eta_{3}>\eta_{2}>\eta_{5}>\eta_{1}$ \\
\hline
\end{tabular}

Table 3. Ordering by the DHPFGGWHM operators.

\begin{tabular}{ccccccc}
\hline Parameter & $s\left(\eta_{\mathbf{1}}\right)$ & $s\left(\eta_{\mathbf{2}}\right)$ & $s\left(\eta_{\mathbf{3}}\right)$ & $s\left(\eta_{\mathbf{4}}\right)$ & $\boldsymbol{s}\left(\eta_{\mathbf{5}}\right)$ & Ordering \\
\hline$\xi=\zeta=0.5$ & 0.5484 & 0.5775 & 0.5431 & 0.6850 & 0.5728 & $\eta_{4}>\eta_{2}>\eta_{5}>\eta_{1}>\eta_{3}$ \\
$\xi=\zeta=1$ & 0.4901 & 0.5176 & 0.4710 & 0.6165 & 0.5256 & $\eta_{4}>\eta_{5}>\eta_{2}>\eta_{1}>\eta_{3}$ \\
$\xi=\zeta=2$ & 0.4349 & 0.4549 & 0.3976 & 0.5240 & 0.4780 & $\eta_{4}>\eta_{5}>\eta_{2}>\eta_{1}>\eta_{3}$ \\
$\xi=\zeta=3$ & 0.4033 & 0.4146 & 0.3563 & 0.4591 & 0.4515 & $\eta_{4}>\eta_{5}>\eta_{2}>\eta_{1}>\eta_{3}$ \\
$\xi=\zeta=4$ & 0.3815 & 0.3854 & 0.3290 & 0.4139 & 0.4344 & $\eta_{5}>\eta_{4}>\eta_{2}>\eta_{1}>\eta_{3}$ \\
$\xi=\zeta=5$ & 0.3653 & 0.3633 & 0.3094 & 0.3817 & 0.4223 & $\eta_{5}>\eta_{4}>\eta_{1}>\eta_{2}>\eta_{3}$ \\
\hline
\end{tabular}

\subsection{Comparative Analysis}

The prominent characteristic of the DHPFGWHM and DHPFGGWHM operators is that they can consider the interrelationship among the DHFNs. Next, we shall compare our developed methods with the dual hesitant Pythagorean fuzzy weighted average (DHPFWA) and dual hesitant Pythagorean fuzzy weighted geometric (DHPFWG) operators [53], with the comparative analysis results listed as follows.

According to Table 1 and attribute weights, the fused values obtained by the DHPFGWA operator are:

$$
\begin{aligned}
& \widetilde{p}_{1}=\operatorname{DHPFWA}\left(\widetilde{p}_{11}, \widetilde{p}_{12}, \widetilde{p}_{13}, \widetilde{p}_{14}\right)=\bigoplus_{j=1}^{4} w_{j} \widetilde{p}_{1 j} \\
& =\left\{\left\{\begin{array}{l}
0.4325,0.4527,0.4522,0.4711,0.4793,0.4967,0.4962,0.5126 \\
0.4580,0.4766,0.4761,0.4936,0.5013,0.5174,0.5170,0.5323
\end{array}\right\},\left\{\begin{array}{l}
0.5872, \\
0.6334
\end{array}\right\}\right\} ;
\end{aligned}
$$




$$
\begin{gathered}
\widetilde{p}_{2}=\operatorname{DHPFWA}\left(\widetilde{p}_{21}, \widetilde{p}_{22}, \widetilde{p}_{23}, \widetilde{p}_{24}\right)=\underset{j=1}{\oplus} w_{j} \widetilde{p}_{2 j} \\
=\{\{0.5005,0.5461,0.5788\},\{0.5476,0.5617,0.5677,0.5823,0.5860,0.6011\}\} ; \\
\widetilde{p}_{3}=\operatorname{DHPFWA}\left(\widetilde{p}_{31}, \widetilde{p}_{32}, \widetilde{p}_{33}, \widetilde{p}_{34}\right)=\underset{j=1}{\oplus} w_{j} \widetilde{p}_{3 j} \\
\left.=\left\{\begin{array}{l}
0.4547,0.4812,0.5178,0.4775,0.5022,0.5364,0.5071,0.5296,0.5610, \\
0.5595,0.5784,0.6051,0.5757,0.5936,0.6190,0.5973,0.6139,0.6376
\end{array}\right\},\left\{\begin{array}{l}
0.5480, \\
0.5704
\end{array}\right\}\right\} ; \\
\widetilde{p}_{4}=\operatorname{DHPFWA}\left(\widetilde{p}_{41}, \widetilde{p}_{42}, \widetilde{p}_{43}, \widetilde{p}_{44}\right)=\underset{j=1}{\oplus} w_{j} \widetilde{p}_{4 j} \\
=\{\{0.6278,0.6347,0.6796,0.6852,0.7529,0.7570\},\{0.3923,0.3997,0.4063\}\} ; \\
\widetilde{p}_{5}=\operatorname{DHPFWA}\left(\widetilde{p}_{51}, \widetilde{p}_{52}, \widetilde{p}_{53}, \widetilde{p}_{54}\right)=\underset{j=1}{\oplus} w_{j} \widetilde{p}_{5 j} \\
\left.=\left\{\begin{array}{l}
0.3434,0.3574,0.3761,0.3936,0.4053,0.4213,0.3763,0.3888,0.4056, \\
0.4214,0.4321,0.4467,0.4172,0.4281,0.4428,0.4568,0.4663,0.4794, \\
0.3531,0.3666,0.3848,0.4017,0.41320 .4287,0.3849,0.3971,0.4134, \\
0.4288,0.4393,0.4535,0.4247,0.4353,0.4497,0.4634,0.4727,0.4855
\end{array}\right\},\{0.4184\}\right\} .
\end{gathered}
$$

Then, based on the score function of the dual hesitant Pythagorean fuzzy elements (DHPFEs), we can obtain the score results of $\widetilde{p}_{i}$ as:

$$
\begin{aligned}
& s\left(\widetilde{p}_{1}\right)=0.4317, s\left(\widetilde{p}_{2}\right)=0.4822, s\left(\widetilde{p}_{3}\right)=0.4977 \\
& s\left(\widetilde{p}_{4}\right)=0.6592, s\left(\widetilde{p}_{5}\right)=0.5005
\end{aligned}
$$

Then, we rank all the suppliers in supply chain management $\eta_{i}(i=1,2,3,4,5)$ in accordance with the scores $s\left(\widetilde{p}_{i}\right)(i=1,2,3,4,5)$ of the overall dual hesitant Pythagorean fuzzy values $\widetilde{p}_{i}(i=1,2, \cdots, 5)$ : $\eta_{4}>\eta_{5}>\eta_{3}>\eta_{2}>\eta_{1}$ and thus the most desirable supplier in supply chain management is obtained, which is $\eta_{4}$.

According to Table 1 and attribute weights, the fused values by the DHPFWG operator are:

$$
\begin{aligned}
& \widetilde{p}_{1}=\operatorname{DHPFWG}\left(\widetilde{p}_{11}, \widetilde{p}_{12}, \widetilde{p}_{13}, \widetilde{p}_{14}\right)=\bigotimes_{j=1}^{4}\left(\widetilde{p}_{1 j}\right)^{w_{j}} \\
& =\left\{\left\{\begin{array}{l}
0.4119,0.4226,0.4452,0.4567,0.4383,0.4496,0.4737,0.4859, \\
0.4356,0.4468,0.4708,0.4829,0.4634,0.4754,0.5009,0.5138
\end{array}\right\},\left\{\begin{array}{l}
0.6574, \\
0.6735
\end{array}\right\}\right\} \\
& \widetilde{p}_{2}=\operatorname{DHPFWG}\left(\widetilde{p}_{21}, \widetilde{p}_{22}, \widetilde{p}_{23}, \widetilde{p}_{24}\right)=\underset{j=1}{\otimes}\left(\widetilde{p}_{2 j}\right)^{w_{j}} \\
& =\{\{0.4086,0.4861,0.5171\},\{0.5694,0.5822,0.6203,0.6311,0.6945,0.7026\}\} ;
\end{aligned}
$$

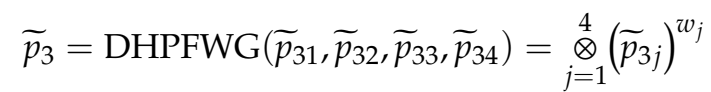

$$
\begin{aligned}
& =\left\{\left\{\begin{array}{l}
0.3931,0.4017,0.4093,0.4335,0.4430,0.4513,0.4677,0.4779,0.4869, \\
0.4224,0.4316,0.4398,0.4658,0.4760,0.4850,0.5026,0.5135,0.5232
\end{array}\right\},\left\{\begin{array}{l}
0.6622, \\
0.7404
\end{array}\right\}\right\} ; \\
& \widetilde{p}_{4}=\operatorname{DHPFWG}\left(\widetilde{p}_{41}, \widetilde{p}_{42}, \widetilde{p}_{43}, \widetilde{p}_{44}\right)=\underset{j=1}{\otimes}\left(\widetilde{p}_{4 j}\right)^{w_{j}} \\
& =\{\{0.6278,0.6347,0.6796,0.6852,0.7529,0.7570\},\{0.3923,0.3997,0.4063\}\} ; \\
& \widetilde{p}_{5}=\operatorname{DHPFWG}\left(\widetilde{p}_{51}, \widetilde{p}_{52}, \widetilde{p}_{53}, \widetilde{p}_{54}\right)=\underset{j=1}{\otimes}\left(\widetilde{p}_{5 j}\right)^{w_{j}} \\
& \left.=\left\{\begin{array}{l}
0.2617,0.2724,0.2811,0.2749,0.2862,0.2952,0.2885,0.3004,0.3099, \\
0.3031,0.3156,0.3256,0.3113,0.3241,0.3344,0.3270,0.3404,0.3512, \\
0.3112,0.3240,0.3342,0.3269,0.3403,0.3511,0.3431,0.3572,0.3686, \\
0.3605,0.3753,0.3872,0.3702,0.3854,0.3976,0.3889,0.4049,0.4177
\end{array}\right\},\{0.4811\}\right\} .
\end{aligned}
$$


Then, based on the score function of the DHPFEs, we can obtain the score results of $\widetilde{p}_{i}$ as:

$$
\begin{aligned}
& s\left(\widetilde{p}_{1}\right)=0.3851, s\left(\widetilde{p}_{2}\right)=0.4099, s\left(\widetilde{p}_{3}\right)=0.3584 \\
& s\left(\widetilde{p}_{4}\right)=0.4939, s\left(\widetilde{p}_{5}\right)=0.4410
\end{aligned}
$$

Then, we rank all the suppliers in supply chain management $\eta_{i}(i=1,2,3,4,5)$ in accordance with the scores $s\left(\widetilde{p}_{i}\right)(i=1,2,3,4,5)$ of the overall dual hesitant Pythagorean fuzzy values $\widetilde{p}_{i}(i=1,2, \cdots, 5)$ : $\eta_{4}>\eta_{5}>\eta_{2}>\eta_{1}>\eta_{3}$ and thus the most desirable supplier in supply chain management is obtained, which is $\eta_{4}$.

According to Table 4, we can easily conclude that the ordering is slightly different and that these are some of the best alternatives. However, our defined operators are mainly characteristic of the advantages that can consider the interrelationship between the arguments being fused into consideration and consider the human hesitance in practical MADM problems. Obviously, the DHPFWA and DHPFWG operators defined by Wei and Lu [53] cannot consider the interrelationship between the arguments being fused. In addition, in a complicated decision-making environment, the decision maker's risk attitude is an important factor to think about, and our methods can do this by altering the parameters $\xi$ and $\zeta$, whereas the DHPFWA and DHPFWG operators presented by Wei and Lu [53] do not have the ability to dynamically adjust to the parameters according to the decision maker's risk attitude, meaning it is difficult to solve risk multiple attribute decision making in real practice.

Table 4. Ordering of the suppliers by the DHPFGGWHM operators.

\begin{tabular}{cc}
\hline Methods & Ordering \\
\hline The DHPFWA operator [53] & $\eta_{4}>\eta_{5}>\eta_{3}>\eta_{2}>\eta_{1}$ \\
The DHPFWG operator [53] & $\eta_{4}>\eta_{5}>\eta_{2}>\eta_{1}>\eta_{3}$ \\
The DHPFGWHM operator & $\eta_{4}>\eta_{5}>\eta_{3}>\eta_{2}>\eta_{1}$ \\
The DHPFGGWHM operator & $\eta_{4}>\eta_{5}>\eta_{2}>\eta_{1}>\eta_{3}$ \\
\hline
\end{tabular}

\section{Conclusions}

Dual hesitant Pythagorean fuzzy numbers have applied the advantages of DHFSs and PFSs. They can flexibly denote decision-making information as well as effectively characterize the reliability of information. Thus, it is meaningful to study MADM problems with DHPFNs. In this paper, based on the generalized Heronian mean operator and generalized geometric Heronian mean operator, we developed some dual hesitant Pythagorean fuzzy Heronian mean aggregation operators: dual hesitant Pythagorean fuzzy generalized weighted Heronian mean (DHPFGWHM) operator and dual hesitant Pythagorean fuzzy generalized geometric weighted Heronian mean (DHPFGGWHM) operator. The significant merits of these defined operators are investigated. Moreover, we have adopted DHPFGWHM and DHPFGGWHM operators to build a decision-making model for MADM problems. In the end, we utilize a concrete instance for suppliers selection in supply chain management to demonstrate our defined model and to testify its accuracy and scientific ability. However, our developed methods can only deal with MADMs with dual hesitant Pythagorean fuzzy information, and it is clear that these operators cannot handle more complicated decision making problems, such as when the sum square of the membership and non-membership is more than 1 . In the future, we shall continue studying MADM problems with the application and extension of the developed operators to other domains $[67,68]$ and proposed more suitable methods [69-75].

Author Contributions: M.T., J.W., J.L., G.W., C.W. and Y.W. conceived and worked together to achieve this work, M.T. and J.W. compiled the computing program by Excel and analyzed the data, J.W. and G.W. wrote the paper. Finally, all the authors have read and approved the final manuscript.

Funding: This work was supported by the University Students' Innovation and Entrepreneurship Training Program Project of Sichuan Normal University under Grant No. 201810636122 and the National Natural Science Foundation of China under Grant No. 71571128. 
Conflicts of Interest: The authors declare no conflict of interest.

\section{References}

1. Zadeh, L.A. Fuzzy Sets. Inf. Control 1965, 8, 338-356.

2. Atanassov, K. Intuitionistic fuzzy sets. Fuzzy Sets Syst. 1986, 20, 87-96. [CrossRef]

3. Zhou, W.; Xu, Z.S. Extended Intuitionistic Fuzzy Sets Based on the Hesitant Fuzzy Membership and their Application in Decision Making with Risk Preference. Int. J. Intell. Syst. 2018, 33, 417-443. [CrossRef]

4. Zhao, F.; Liu, H.; Fan, J.; Chen, C.W.; Lan, R.; Li, N. Intuitionistic fuzzy set approach to multi-objective evolutionary clustering with multiple spatial information for image segmentation. Neurocomput. 2018, 312, 296-309. [CrossRef]

5. Zhang, Z.M. Geometric Bonferroni means of interval-valued intuitionistic fuzzy numbers and their application to multiple attribute group decision making. Neural Comput. Appl. 2018, 29, 1139-1154. [CrossRef]

6. Zhang, G.; Zhang, Z.; Kong, H. Some Normal Intuitionistic Fuzzy Heronian Mean Operators Using Hamacher Operation and Their Application. Symmetry 2018, 10, 199. [CrossRef]

7. Li, Z.; Gao, H.; Wei, G. Methods for Multiple Attribute Group Decision Making Based on Intuitionistic Fuzzy Dombi Hamy Mean Operators. Symmetry 2018, 10, 574. [CrossRef]

8. Wei, G.W. TODIM Method for Picture Fuzzy Multiple Attribute Decision Making. Informatica 2018, 29, 555-566. [CrossRef]

9. Wang, J.; Wei, G.W.; Lu, M. TODIM Method for Multiple Attribute Group Decision Making under 2-Tuple Linguistic Neutrosophic Environment. Symmetry 2018, 10, 486. [CrossRef]

10. Zhai, Y.L.; Xu, Z.S.; Liao, H.C. Measures of Probabilistic Interval-Valued Intuitionistic Hesitant Fuzzy Sets and the Application in Reducing Excessive Medical Examinations. IEEE Trans. Fuzzy Syst. 2018, 26, 1651-1670.

11. Li, Z.; Wei, G.; Lu, M. Pythagorean Fuzzy Hamy Mean Operators in Multiple Attribute Group Decision Making and Their Application to Supplier Selection. Symmetry 2018, 10, 505. [CrossRef]

12. Wu, L.; Wei, G.; Gao, H.; Wei, Y. Some Interval-Valued Intuitionistic Fuzzy Dombi Hamy Mean Operators and Their Application for Evaluating the Elderly Tourism Service Quality in Tourism Destination. Mathematics 2018, 6, 294. [CrossRef]

13. Wang, J.; Gao, H.; Wei, G.; Wei, Y. Methods for Multiple-Attribute Group Decision Making with q-Rung Interval-Valued Orthopair Fuzzy Information and Their Applications to the Selection of Green Suppliers. Symmetry 2019, 11, 56. [CrossRef]

14. Wei, G.W.; Zhang, Z.P. Some single-valued neutrosophic Bonferroni power aggregation operators in multiple attribute decision making. J. Ambient. Intell. Humaniz. Comput. 2019, 10, 863-882. [CrossRef]

15. Zhang, S.; Gao, H.; Wei, G.; Wei, Y.; Wei, C. Evaluation Based on Distance from Average Solution Method for Multiple Criteria Group Decision Making under Picture 2-Tuple Linguistic Environment. Mathematics 2019, 7, 243. [CrossRef]

16. Wei, G.-W. Pythagorean Fuzzy Hamacher Power Aggregation Operators in Multiple Attribute Decision Making. Fundam. Inform. 2019, 166, 57-85. [CrossRef]

17. Xu, Z.S. Intuitionistic fuzzy aggregation operators. IEEE Trans. Fuzzy Syst. 2007, 15, 1179-1187.

18. Xu, Z.; Yager, R.R. Some geometric aggregation operators based on intuitionistic fuzzy sets. Int. J. Gen. Syst. 2006, 35, 417-433. [CrossRef]

19. Hung, W.L.; Yang, M.S. Similarity measures of intuitionistic fuzzy sets based on L-p metric. Int. J. Approx. Reason. 2007, 46, 120-136. [CrossRef]

20. Park, J.H.; Lim, K.M.; Park, J.S.; Kwun, Y.C. Distances between Interval-valued Intuitionistic Fuzzy Sets. In Proceedings of the International Symposium on Nonlinear Dynamics, Shanghai, China, 27-30 October 2007.

21. Wei, G.-W. Maximizing deviation method for multiple attribute decision making in intuitionistic fuzzy setting. Knowl. Based Syst. 2008, 21, 833-836. [CrossRef]

22. Hung, C.C.; Chen, L.H.; Ao, S.I. A Fuzzy TOPSIS Decision Making Model with Entropy Weight under Intuitionistic Fuzzy Environment. In Proceedings of the International of Multi Conference of Engineers and Computer Scientist (IMECS), Hong Kong, China, 18-20 March 2009.

23. Luo, Y.J. IEEE, Projection Method for Multiple Attribute Decision Making with Uncertain Attribute Weights under Intuitionistic Fuzzy Environment. In Proceedings of the Chinese Control and Decision Conference, Guilin, China, 17-19 June 2009; pp. 2945-2948. 
24. Ye, J. Fuzzy cross entropy of interval-valued intuitionistic fuzzy sets and its optimal decision-making method based on the weights of alternatives. Syst. Appl. 2011, 38, 6179-6183. [CrossRef]

25. Zhang, Z.M. Interval-Valued Intuitionistic Hesitant Fuzzy Aggregation Operators and Their Application in Group Decision-Making. J. Appl. Math. 2013, 2013. [CrossRef]

26. Liao, H.C.; Xu, Z.S. Intuitionistic Fuzzy Hybrid Weighted Aggregation Operators. Int. J. Intell. Syst. 2014, 29, 971-993. [CrossRef]

27. Liu, X.Y.; Ju, Y.B.; Yang, S.H. Hesitant intuitionistic fuzzy linguistic aggregation operators and their applications to multiple attribute decision making. J. Intell. Fuzzy Syst. 2014, 27, 1187-1201.

28. Peng, J.-J.; Wang, J.-Q.; Wang, J.; Chen, X.-H. Multicriteria Decision-Making Approach with Hesitant Interval-Valued Intuitionistic Fuzzy Sets. Sci. J. 2014, 2014, 1-22. [CrossRef] [PubMed]

29. Chen, J.J.; Huang, X.J. Hesitant triangular intuitionistic fuzzy information and its application to multi-attribute decision making problem. J. Nonlinear Sci. Appl. 2017, 10, 1012-1029. [CrossRef]

30. Leśniak, A.; Kubek, D.; Plebankiewicz, E.; Zima, K.; Belniak, S. Fuzzy AHP Application for Supporting Contractors' Bidding Decision. Symmetry 2018, 10, 642. [CrossRef]

31. Adeel, A.; Akram, M.; Ahmed, I.; Nazar, K. Novel m-Polar Fuzzy Linguistic ELECTRE-I Method for Group Decision-Making. Symmetry 2019, 11, 471. [CrossRef]

32. Turskis, Z.; Goranin, N.; Nurusheva, A.; Boranbayev, S. A Fuzzy WASPAS-Based Approach to Determine Critical Information Infrastructures of EU Sustainable Development. Sustainability 2019, 11, 424. [CrossRef]

33. Ziemba, P.; Becker, J. Analysis of the Digital Divide Using Fuzzy Forecasting. Symmetry 2019, 11, 166. [CrossRef]

34. Hu, C.-K.; Liu, F.-B. A Hybrid Fuzzy DEA/AHP Methodology for Ranking Units in a Fuzzy Environment. Symmetry 2017, 9, 273. [CrossRef]

35. Ziemba, P.; Jankowski, J.; Wątróbski, J. Online Comparison System with Certain and Uncertain Criteria Based on Multi-criteria Decision Analysis Method. In Text, Speech and Dialogue; Springer Nature: Heidelberger, Germany, 2017; Volume 10449, pp. 579-589.

36. Diouf, M.; Kwak, C. Fuzzy AHP, DEA, and Managerial Analysis for Supplier Selection and Development; From the Perspective of Open Innovation. Sustainability 2018, 10, 3779. [CrossRef]

37. Dong, J.; Li, R.; Huang, H. Performance Evaluation of Residential Demand Response Based on a Modified Fuzzy VIKOR and Scalable Computing Method. Energies 2018, 11, 1097. [CrossRef]

38. Kim, J.; Kim, J. Optimal Portfolio for LNG Importation in Korea Using a Two-Step Portfolio Model and a Fuzzy Analytic Hierarchy Process. Energies 2018, 11, 3049. [CrossRef]

39. Chou, Y.C.; Yen, H.Y.; Dang, V.T.; Sun, C.C. Assessing the Human Resource in Science and Technology for Asian Countries: Application of Fuzzy AHP and Fuzzy TOPSIS. Symmetry 2019, 11, 251. [CrossRef]

40. Yager, R.R. Pythagorean Fuzzy Subsets. In Proceedings of the Joint IFSA World Congress and NAFIPS Annual Meeting (IFSA/NAFIPS), Edmonton, Alberta, Canada, 24-28 June 2013; pp. 57-61.

41. Yager, R.R. Pythagorean Membership Grades in Multicriteria Decision Making. IEEE Trans. Syst. 2014, 22, 958-965. [CrossRef]

42. Zhang, X.L.; Xu, Z.S. Extension of TOPSIS to Multiple Criteria Decision Making with Pythagorean Fuzzy Sets. Int. J. Intell. Syst. 2014, 29, 1061-1078. [CrossRef]

43. Peng, X.; Yang, Y. Some Results for Pythagorean Fuzzy Sets. Int. J. Intell. Syst. 2015, 30, 1133-1160. [CrossRef]

44. Reformat, M.Z.; Yager, R.R. Suggesting Recommendations Using Pythagorean Fuzzy Sets illustrated Using Netflix Movie Data. In Communications in Computer and Information Science; Springer Nature: Basingstoke, UK, 2014; Volume 442, pp. 546-556.

45. Gou, X.J.; Xu, Z.S.; Ren, P.J. The Properties of Continuous Pythagorean Fuzzy Information. Int. J. Intell. Syst. 2016, 31, 401-424. [CrossRef]

46. Garg, H. A New Generalized Pythagorean Fuzzy Information Aggregation Using Einstein Operations and Its Application to Decision Making. Int. J. Intell. Syst. 2016, 31, 886-920. [CrossRef]

47. Zeng, S.Z.; Chen, J.P.; Li, X.S. A Hybrid Method for Pythagorean Fuzzy Multiple-Criteria Decision Making. Int. J. Inf. Technol. Decis. Mak. 2016, 15, 403-422. [CrossRef]

48. Ren, P.J.; Xu, Z.S.; Gou, X.J. Pythagorean fuzzy TODIM approach to multi-criteria decision making. Appl. Soft Comput. 2016, 42, 246-259. [CrossRef]

49. Liang, D.; Zhang, Y.; Xu, Z.; Darko, A.P. Pythagorean fuzzy Bonferroni mean aggregation operator and its accelerative calculating algorithm with the multithreading. Int. J. Intell. Syst. 2018, 33, 615-633. [CrossRef] 
50. Liang, D.; Xu, Z.; Darko, A.P. Projection Model for Fusing the Information of Pythagorean Fuzzy Multicriteria Group Decision Making Based on Geometric Bonferroni Mean. Int. J. Intell. Syst. 2017, 32, 966-987. [CrossRef]

51. Zhu, B.; Xu, Z.S.; Xia, M.M. Dual Hesitant Fuzzy Sets. J. Appl. Math. 2012, 2012. [CrossRef]

52. Wang, H.J.; Zhao, X.F.; Wei, G.W. Dual hesitant fuzzy aggregation operators in multiple attribute decision making. J. Intell. Fuzzy Syst. 2014, 26, 2281-2290.

53. Wei, G.; Lu, M. Dual hesitant pythagorean fuzzy Hamacher aggregation operators in multiple attribute decision making. Arch. Control. Sci. 2017, 27, 365-395. [CrossRef]

54. Yu, D.J. Intuitionistic fuzzy geometric Heronian mean aggregation operators. Appl. Soft Comput. 2013, 13, 1235-1246. [CrossRef]

55. Fan, C.; Ye, J.; Feng, S.; Fan, E.; Hu, K. Multi-Criteria Decision-Making Method Using Heronian Mean Operators under a Bipolar Neutrosophic Environment. Mathematics 2019, 7, 97. [CrossRef]

56. Zang, Y.Q.; Zhao, X.D.; Li, S.Y. Interval-Valued Dual Hesitant Fuzzy Heronian Mean Aggregation Operators and their Application to Multi-Attribute Decision Making. Int. J. Comput. Intell. Appl. 2018, 17, 1850005. [CrossRef]

57. Wei, G.W.; Lu, M.; Gao, H. Picture fuzzy heronian mean aggregation operators in multiple attribute decision making. Int. J. Knowl. Intell. Eng. Syst. 2018, 22, 167-175. [CrossRef]

58. Wang, H.H.; Ju, Y.B.; Liu, P.D.; Ju, D.W.; Liu, Z.M. Some trapezoidal interval type-2 fuzzy Heronian mean operators and their application in multiple attribute group decision making. J. Intell. Fuzzy Syst. 2018, 35, 2323-2337. [CrossRef]

59. Fan, C.; Ye, J. Heronian Mean Operator of Linguistic Neutrosophic Cubic Numbers and Their Multiple Attribute Decision-Making Methods. Math. Probl. Eng. 2018, 2018, 1-13. [CrossRef]

60. Liu, P.; Liu, Z.; Zhang, X. Some intuitionistic uncertain linguistic Heronian mean operators and their application to group decision making. Appl. Math. Comput. 2014, 230, 570-586. [CrossRef]

61. Yu, S.-M.; Zhou, H.; Chen, X.-H.; Wang, J.-Q. A Multi-Criteria Decision-Making Method Based on Heronian Mean Operators Under a Linguistic Hesitant Fuzzy Environment. Asia-Pacific J. Oper. Res. 2015, 32, 1550035. [CrossRef]

62. Li, Y.H.; Liu, P.D.; Chen, Y.B. Some Single Valued Neutrosophic Number Heronian Mean Operators and Their Application in Multiple Attribute Group Decision Making. Informatica 2016, 27, 85-110. [CrossRef]

63. Wei, G.; Gao, H.; Wei, Y. Some q-rung orthopair fuzzy Heronian mean operators in multiple attribute decision making. Int. J. Intell. Syst. 2018, 33, 1426-1458. [CrossRef]

64. Li, L.; Zhang, R.T.; Wang, J.; Shang, X.P. Some q-rung orthopair linguistic Heronian mean operators with their application to multi-attribute group decision making. Arch. Control Sci. 2018, 28, 551-583.

65. Beliakov, G.P.; Calvo, A.T. Aggregation Functions: A Guide for Practitioners; Springer: Heidelberg, Germany, 2007.

66. Xu, Y.; Shang, X.; Wang, J.; Wu, W.; Huang, H. Some q-Rung Dual Hesitant Fuzzy Heronian Mean Operators with Their Application to Multiple Attribute Group Decision-Making. Symmetry 2018, 10, 472. [CrossRef]

67. Wei, Y.; Yu, Q.; Liu, J.; Cao, Y. Hot money and China's stock market volatility: Further evidence using the GARCH-MIDAS model. Phys. A: Stat. Mech. Appl. 2018, 492, 923-930. [CrossRef]

68. Wei, Y.; Liu, J.; Lai, X.; Hu, Y. Which determinant is the most informative in forecasting crude oil market volatility: Fundamental, speculation, or uncertainty? Energy Economics 2017, 68, 141-150. [CrossRef]

69. Wang, R.; Wang, J.; Gao, H.; Wei, G.W. Methods for MADM with Picture Fuzzy Muirhead Mean Operators and Their Application for Evaluating the Financial Investment Risk. Symmetry 2019, 11, 6. [CrossRef]

70. Wang, J.; Wei, G.W.; Wei, Y. Models for Green Supplier Selection with Some 2-Tuple Linguistic Neutrosophic Number Bonferroni Mean Operators. Symmetry 2018, 10, 131. [CrossRef]

71. Gao, H. Pythagorean fuzzy Hamacher Prioritized aggregation operators in multiple attribute decision making. J. Intell. Fuzzy Syst. 2018, 35, 2229-2245. [CrossRef]

72. Deng, X.M.; Wei, G.W.; Gao, H.; Wang, J. Models for Safety Assessment of Construction Project With Some 2-Tuple Linguistic Pythagorean Fuzzy Bonferroni Mean Operators. IEEE Access 2018, 6, 52105-52137. [CrossRef]

73. Deng, X.M.; Wang, J.; Wei, G.W.; Lu, M. Models for Multiple Attribute Decision Making with Some 2-Tuple Linguistic Pythagorean Fuzzy Hamy Mean Operators. Mathematics 2018, 6, 11. [CrossRef] 
74. Wei, G.W.; Garg, H.; Gao, H.; Wei, C. Interval-Valued Pythagorean Fuzzy Maclaurin Symmetric Mean Operators in Multiple Attribute Decision Making. IEEE Access 2018, 6, 67866-67884. [CrossRef]

75. Li, Z.X.; Wei, G.W.; Gao, H. Methods for Multiple Attribute Decision Making with Interval-Valued Pythagorean Fuzzy Information. Mathematics 2018, 6, 228. [CrossRef]

(C) 2019 by the authors. Licensee MDPI, Basel, Switzerland. This article is an open access article distributed under the terms and conditions of the Creative Commons Attribution (CC BY) license (http://creativecommons.org/licenses/by/4.0/). 